\title{
Monthly Runoff Forecasting Using Variational Mode Decomposition Coupled with Grey Wolf Optimizer Based Long Short-term Memory Neural Networks
}

Bao-Jian Li ( $\sim$ libaojian@ncwu.edu.cn )

North China University of Water Resources and Electric Power

Guo-Liang Sun

North China University of Water Resources and Electric Power

Yan Liu

Zhengzhou University of Light Industry

Wen-Chuan Wang

North China University of Water Resources and Electric Power

Xu-Dong Huang

North China University of Water Resources and Electric Power

\section{Research Article}

Keywords: variational mode decomposition, long short-term memory neural networks, gray wolf optimizer, monthly runoff forecasting

Posted Date: January 17th, 2022

DOI: https://doi.org/10.21203/rs.3.rs-1018857/v1

License: (9) (1) This work is licensed under a Creative Commons Attribution 4.0 International License.

Read Full License 


\title{
Monthly Runoff Forecasting Using Variational Mode Decomposition
}

\section{Coupled with Grey Wolf Optimizer Based Long Short-term Memory}

\author{
Neural Networks
}

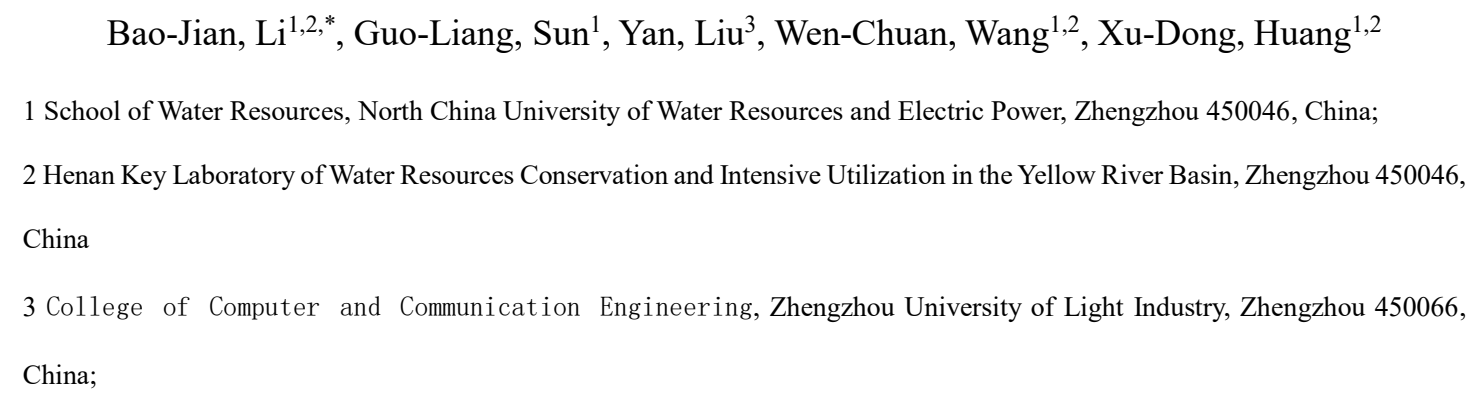

Abstract: Accurate and reliable monthly runoff forecasting plays an important role in making full use of water resources. In recent years, long short-term memory neural networks (LSTM), as a deep learning technology, has been successfully applied in forecasting monthly runoff. However, the hyperparameters of LSTM is predetermined, which has a significant influence on model performance. In this study, given decomposition of monthly runoff series may provide more accurate predication revealed by many previous studies, a hybrid model, namely VMD-GWO-LSTM, has been proposed for monthly runoff forecasting. The proposed hybrid model is comprised of two main components, namely variational mode decomposition (VMD) coupled with grey wolf optimizer (GWO) based LSTM. First, VMD is utilized to decompose raw monthly runoff series into several subsequences. Second, GWO is implemented to optimize the hyperparameters of LSTM for each subsequence on condition that the inputs are determined. Finally, the total output of all subsequences is aggregated as final forecast result. Four quantitative indexes are employed to evaluate the model performance. The proposed model is demonstrated using monthly runoff series data derived from two reservoirs in China's Pearl River system. To identify the feasibility and superiority of the proposed model, back propagation neural networks (BPNN), support vector machine (SVM), LSTM, EMD-LSTM, VMD-LSTM and GWO-LSTM are also utilized for comparison. The results indicate that the proposed hybrid model can yield best forecast accuracy among these models, making it a promising new method for monthly runoff forecasting.

Keywords: variational mode decomposition, long short-term memory neural networks, gray wolf optimizer, monthly runoff forecasting

\footnotetext{
*corresponding author

E-mail address: libaojian@ncwu.edu.cn (B.-J. Li).
} 


\section{Introduction}

Accurate and reliable monthly runoff forecasting plays an important role in water resources management, such as water supply (Şen, 2021), hydroelectric generation (Feng et al., 2020a) and ecological restoration (Feng et al., 2018). Generally, existing methods can be approximately partitioned into data-driven (Feng et al., 2021; Liao et al., 2020; Wang et al., 2015), and physical-based models (Dakhlalla and Parajuli, 2016; Liao et al., 2016; Madsen, 2003). The data-based models can simulate the relationship between input and output without regard to complex mechanisms of runoff generation (Niu et al., 2019). As a contrast, the physical-based models take into account specific physical process and demand mass data such as underlying surface conditions, human activity influence and climate change which are not easily collected (Feng and Niu, 2021). Unlike the physical-based models, the data-based models demand less data and can afford satisfactory forecast results. As a typical representative of data-based models, artificial neural networks (ANN) has been widely and successfully utilized in hydrology-related areas, for instance, precipitation forecasting (Nourani et al., 2009), runoff forecasting (Shoaib et al., 2018), water level forecasting (Seo et al., 2015), etc. In the past few decades, numerous ANN architectures and algorithms have been investigated in hydrological time series forecasting (ASCE-Task-Committee, 2000).

Long short-term memory neural networks (LSTM) proposed by Hochreiter and Schmidhuber (Hochreiter and Schmidhuber, 1997) is a special kind of recurrent neural network (RNN) and has merits of fast convergence and good nonlinear predictive capability. To avoid the problems of training long sequences and vanishing gradients faced by the traditional RNN, LSTM implements constant error flow via constant error carrousels within special memory cells. Referred to LSTM, many literatures have been studied in hydrological time series forecasting ( $\mathrm{Lv}$ et al., 2020; Ni et al., 2020; Wang et al., 2021). Nevertheless, the hyperparameters of LSTM are predetermined, which has a certain impact on forecast accuracy. In general, there are two main methods to improve the forecast accuracy in the previous studies. The first is to combine decomposition algorithms to decompose original time series data into several subcomponents, employ LSTM to simulate each subcomponent, and aggregate the result of each subcomponent as the final result (Lv et al., 2020). Zuo et al. (Zuo et al., 2020), for example, proposed a single-model forecasting based on VMD and LSTM to predict daily streamflow 1-7 days ahead, and investigated the robustness and efficiency of the proposed model for forecasting highly nonstationary and nonlinear streamflow. The Second is to utilize optimization algorithms to optimize the 
hyperparameters of the LSTM (ElSaid et al., 2018). Yuan et al. (Yuan et al., 2018), for example, used ant lion optimizer (ALO) to calibrate the parameters of the LSTM, and verified its effectiveness with the historical monthly runoff of the Astor River Basin. At present, there are several commonly used decomposition algorithms (Colominas et al., 2014; Roushangar et al., 2021; Shahid et al., 2020), for instance, wavelet decomposition, empirical mode decomposition (EMD) and VMD. The optimization algorithms, such as particle swarm optimization and ant colony optimization, can be seen in the literature as optimizing the parameters of neural networks (Wan et al., 2017; Yu et al., 2008). In this study, both methods are considered.

Variational mode decomposition (VMD) (Dragomiretskiy and Zosso, 2014) is an entirely nonrecursive variational model that can extract modes concurrently. Via VMD, a signal can be decomposed into a sequence of subcomponents with different frequency bands and time resolutions (Fang et al., 2019). Compared to empirical mode decomposition (EMD), VMD is capable of separating tones of similar frequencies. VMD has been widely applied in many research fields, such as fault diagnosis (Zhang et al., 2017), signal processing (Wang et al., 2017), wind speed monitoring (Liu et al., 2018) and hydrological time series forecasting (Feng et al., 2020b; Li et al., 2021; Sibtain et al., 2021). In this study, VMD was selected as a data preprocessing tool to decompose monthly run off series. In recent years, an emerging swarm intelligence algorithm called grey wolf optimizer (GWO) has been proposed, which imitates the social hierarchy and hunting behavior of grey wolves (Mirjalili et al., 2014). With its strong robustness and searching ability in solving optimization problem, GWO has been widely and successfully applied in many fields, like model parameter calibration (Tikhamarine et al., 2020), reservoir operation (Niu et al., 2021) and optimal power dispatch (Nuaekaew et al., 2017). In view of its strong robustness and searching ability, GWO can be adopted to optimize the hyperparameters of LSTM. In this paper, a hybrid model, referred to as VMD-GWO-LSTM, is proposed for monthly runoff forecasting. According to the monthly runoff series of two real-world hydropower reservoirs in China, the proposed method is certified to be feasible. The novel contributions of this study can be stated as follows. (1) To decrease modeling difficulty, VMD is adopted to decompose monthly runoff series into several simple subcomponents. (2) For each subcomponent, the input-output relationships are identified by LSTM and the GWO method is employed to optimize the hyperparameters of LSTM. (3) The results of case study indicate that, compared to several traditional models, the proposed hybrid method VMD-GWO-LSTM can yield better 

forecast monthly runoff, demonstrating this study has the potential to refill this gap.

The rest of this work is organized as follow: Section 2 describes the details of the proposed approach; in Section 3, the proposed method is utilized to forecast the monthly runoff of two reservoirs; finally, the conclusions are summarized.

\section{Methodology}

\subsection{Variational mode decomposition}

VMD is a novel variational method which can non-recursively decompose a nonstationary signal into a given number of mode functions and each individual mode is compact around its center frequency (Dragomiretskiy and Zosso, 2014). To obtain each mode and its center frequency, a constrained variational problem can be expressed as follows:

$$
\left\{\begin{array}{l}
\min _{\left\{u_{k}(t),\left\{\omega_{k}(t)\right\}\right.}\left\{\sum_{k}\left\|\partial_{t}\left[\left(\delta(t)+\frac{j}{\pi t}\right) * u_{k}(t)\right] e^{-j \omega_{k} t}\right\|_{2}^{2}\right\} \\
\text { s.t. } \sum_{k} u_{k}(t)=f(t)
\end{array}\right.
$$

where $t$ is the time step; $u_{k}(t)$ and $\omega_{k}(t)$ denote $k$-th mode and its corresponding center frequency, respectively; $\delta(t)$ is the Dirac distribution, * denotes the convolution calculation and $f(t)$ denotes the $t$-th data of the input signal.

In order to facilitate solution, the quadratic penalty factor $\alpha$ and the Lagrangian multiplier $\lambda$ are introduced to transform the constrained variational problem into an unconstrained variational problem. Hence, the augmented Lagrangian structure can be expressed as follows:

$$
L\left(\left\{u_{k}\right\},\left\{\omega_{k}\right\}, \lambda\right)=\alpha \sum_{k}\left\|\partial_{t}\left[\left(\delta(t)+\frac{j}{\pi t}\right) * u_{k}(t)\right] e^{-j \omega_{k} t}\right\|_{2}^{2}+\left\|f(t)-\sum_{k} u_{k}(t)\right\|_{2}^{2}+\left\langle\lambda(t), f(t)-\sum_{k} u_{k}(t)\right\rangle
$$

where $\langle\cdot\rangle$ represents the inner product operation.

The Eq. (2) can then be solved by the alternating direction method of multiplier (ADMM) to get the saddle point of the augmented Lagrangian function. In the ADMM, the variables $\left(\hat{u}_{k}^{n+1}, \omega_{k}^{n+1}\right.$ and $\left.\hat{\lambda}^{n+1}\right)$ are continuously updated to optimize each modal component. And thus the optimal individual mode $u_{k}$ and the corresponding center frequency $\omega_{k}$ can be obtained by the iterative equation expressed as follows:

$$
\hat{u}_{k}^{n+1}(\omega)=\frac{\hat{f}(\omega)-\sum_{i \neq k} \hat{u}_{i}(\omega)+\frac{\hat{\lambda}(\omega)}{2}}{1+2 \alpha\left(\omega-\omega_{k}\right)^{2}}
$$




$$
\begin{gathered}
\omega_{k}^{n+1}=\frac{\int_{0}^{\infty} \omega\left|\hat{u}_{k}(\omega)\right|^{2} d \omega}{\int_{0}^{\infty}\left|\hat{u}_{k}(\omega)\right|^{2} d \omega} \\
\hat{\lambda}^{n+1}(\omega)=\hat{\lambda}^{n}(\omega)+\tau\left(\hat{f}(\omega)-\sum_{k} \hat{u}_{k}^{n+1}(\omega)\right)
\end{gathered}
$$

115 where $\mathrm{n}$ is the iteration number; $\tau$ is the iterative factor; $\hat{f}(w), \hat{u}_{i}(w), \hat{\lambda}(w)$ and $\hat{u}_{k}^{n+1}$ denote the

116 Fourier transforms of $f(t), u_{i}(t), \lambda(t)$ and $\hat{u}_{k}^{n+1}(t)$, respectively.

\section{2 long short-term memory neural networks}

118 As a type of deep learning neural networks, LSTM was proposed to overcome the gradient vanishing/exploding problem faced by traditional RNN (Hochreiter and Schmidhuber, 1997). LSTM takes place of the conventional hidden unit with a memory cell and contains multiple memory blocks of which each include three gates: input gate, forget gate and output gate and at least a memory cell. By LSTM, information from the three gates can be added or deleted to the memory cell state. Based on the previous state, current memory and current input, the LSTM owns the ability to decide which cells are restrained and promoted and on the basis of the three gates what information is saved and forgotten during the training process (Altan et al., 2021). The structure of LSTM is shown in Fig. 1. For the three gates: the multiplicative input gate unit is employed to recognize new information that can be gathered in the cell; the multiplicative output gate unit is utilized to compute the information that can be propagated to the network; the multiplicative forget gate unit is used to decide whether the last status of the cell can be forgotten (Li et al., 2018).

\section{Insert Here Fig. 1}

131 The calculation of the three gates and cell state can be generally expressed as follows:

$$
\begin{aligned}
& f_{t}=\sigma\left(W_{f} \cdot\left[h_{t-1}, x_{t}\right]+b_{f}\right) \\
& i_{t}=\sigma\left(W_{i} \cdot\left[h_{t-1}, x_{t}\right]+b_{i}\right) \\
& \varepsilon_{t}=\tanh \left(W_{c} \cdot\left[h_{t-1}, x_{t}\right]+b_{c}\right) \\
& c_{t}=f_{t} \mathrm{e} c_{t-1}+i_{t} \mathrm{e} \varepsilon_{t} \\
& o_{t}=\sigma\left(W_{o} \mathrm{e}\left[h_{t-1}, x_{t}\right]+b_{0}\right) \\
& h_{t}=o_{t} \cdot \tanh \left(c_{t}\right)
\end{aligned}
$$

where $f_{t}, i_{t}, o_{t}$ denote the output of the forget gate, input gate and output gate, respectively; $E_{t}$ is 
which usually requires a certain amount of practical experience to manually select and optimize the hyperparameters. Therefore, for convenience, automatic algorithmic approaches with ability to converge faster and gain an optimal/ near optimal solution within an acceptable time can be employed to enhance the performance of LSTM (Nakisa et al., 2018).

\subsection{Grey wolf optimizer}

143 The GWO algorithm is a novel swarm intelligent optimization algorithm that simulates the leadership

144 hierarchy and predation strategy of grey wolves (Mirjalili et al., 2014). Grey wolves possess the very strict 145 social dominant hierarchy, which can be divided into four categories including alpha wolf $(\alpha)$, beta wolf $(\beta)$, delta wolf $(\delta)$ and omega wolf $(\omega)$. The alpha dominates the whole wolf pack and is responsible for making decisions. Beta wolves are subordinate to the alpha in the hierarchy, but command delta and omega wolves as well. Meanwhile, alpha wolves are also responsible for coordinating alpha wolves to make decisions. Delta wolves obey the orders of alpha and beta wolves and are responsible for the detection and guarding of the wolf pack. The omega is the lowest ranking and omega wolves play a vital role in the balance of internal relationships among the pack. The hunting process of grey wolves can be divided into three stages: (i) tracking, chasing and approaching prey; (ii) hunting, surrounding and intruding the prey until it stops moving; (iii) attacking the prey (Mirjalili et al., 2014). The optimal solution is obtained by updating and evolving the positions of wolves, and gradually approaching the prey. The GWO algorithm can be roughly described as follows. follows:

$$
\begin{gathered}
\stackrel{\mathrm{r}}{D}=\left|\stackrel{\mathrm{r}}{C} \cdot \stackrel{\mathrm{r}}{X}{ }_{p}(t)-\stackrel{\mathrm{r}}{X}(t)\right| \\
\stackrel{\mathrm{I}}{X}(t+1)=\stackrel{\mathrm{r}}{X}(t)-\stackrel{\text { I }}{A} \cdot \stackrel{\mathrm{r}}{D} \\
\stackrel{\mathrm{I}}{A}=2 \stackrel{\mathrm{r}}{a} \cdot \stackrel{\mathrm{r}}{r}_{1}-\stackrel{\mathrm{r}}{a} \\
\stackrel{\mathrm{I}}{\mathrm{C}}=2 \cdot \stackrel{\mathrm{r}}{r_{2}}
\end{gathered}
$$

where $t$ is the current iteration; $\stackrel{\mathrm{r}}{D}$ is the distance between the grey wolf and the prey; $\stackrel{\mathrm{r}}{X_{P}}(t)$ is the position vector of the $t$-th prey; $\stackrel{+}{X}(t)$ is the position vector of the $t$-th grey wolf; $\stackrel{\mathrm{I}}{A}$ and $\stackrel{\mathrm{I}}{C}$ are

164 coefficient vectors; $\stackrel{r_{1}}{r}$ and $\stackrel{\stackrel{r}{r}}{r_{2}}$ are random numbers in $[0,1] ; \stackrel{\stackrel{r}{a}}{\text { is }}$ transition parameter and linearly 

better knowledge about the potential position of prey. Hence, the formulas are given as follows which other wolves should be obeyed to update their positions:

$$
\stackrel{\mathrm{r}}{X}(t+1)=\frac{\stackrel{1}{X}_{1}+\stackrel{1}{X}_{2}+\stackrel{1}{X}_{3}}{3}
$$

Finally, attacking prey is executed. In order to simulate being close to the prey, the value of $\stackrel{1}{a}$ is decreased linearly and correspondingly the fluctuation range of $\stackrel{1}{A}$ is also decreased within the interval of [-2a, 2a]. When $\stackrel{\mathrm{t}}{A}$ ranging in $[-1,1]$, the next position of a grey wolf in any position is between its current position and the position of the prey (Mirjalili et al., 2014). And thus the attack on the prey can be realized.

\subsection{Hybrid model for monthly runoff forecasting}

Hydrological time series with the characteristics of nonlinearity and nonstationarity are usually related to various factors, including climate change and variation of underlying surface conditions (Ji et al., 2014; Xu et al., 2017). To improve forecast accuracy of monthly runoff forecasting, a hybrid model shortened to VMD-GWO-LSTM is proposed and illustrated in Fig.2. Firstly, VMD is utilized to decompose the original complex runoff time series into several relatively simple subsequences. Secondly, LSTM of which the hyperparameters are optimized by GWO, is employed to simulated each subsequence. Finally, the simulation results of all subsequences are aggregated as the final forecast result. The main procedure can be described as follows:

Step 1: Data preprocessing. Utilize VMD to decompose the original runoff sequence to obtain $K$ subsequences with different frequencies. All subsequences that are divided into calibration and validation data are normalized to $[-1,1]$. 
variables of each subsequence for the LSTM model.

Step 3: Hyperparameter optimization. The GWO algorithm of which root-mean-squared error (RMSE) is selected as an optimization criterion, is carried out independently to optimize the hyperparameters of the LSTM model for simulating each subsequence. In this study, the optimal parameters of the number of hidden layer neurons, the number of epochs and the learning rate of the LSTM model are searched.

Step 4: Aggregation. The forecast results of all subsequences are arithmetically aggrated as the final forecast results.

\subsection{Evolution index}

Insert Here Fig. 2

Selecting appropriate evaluation criteria is essential for utilizing multi-criteria analysis to validate model performance (Modarres, 2009). In this section, four evolution indexes, namely RMSE, mean absolute percentage error (MAPE), coefficient of correlation (R) and Nash-Sutcliffe efficiency coefficient (CE) are employed. Generally, the smaller RMSE and MAPE and the higher R and CE, the better model performance. These indexes as listed as below:

$$
R M S E=\sqrt{\frac{1}{n} \sum_{i=1}^{n}\left(Q_{i}-\hat{Q}_{i}\right)^{2}}
$$

$$
\text { MAPE }=\frac{1}{n} \sum_{i=1}^{n}\left|\frac{Q_{i}-\hat{Q}_{i}}{Q_{i}}\right| \times 100
$$

$$
R=\frac{\sum_{i=1}^{n}\left(Q_{i}-\bar{Q}_{i}\right)\left(\hat{Q}_{i}-\overline{\hat{Q}}_{i}\right)}{\sqrt{\sum_{i=1}^{n}\left(Q_{i}-\bar{Q}_{i}\right)^{2} \sum_{i=1}^{n}\left(\hat{Q}_{i}-\overline{\hat{Q}}\right)^{2}}}
$$

$$
C E=1-\frac{\sum_{i=1}^{n}\left(Q_{i}-\bar{Q}_{i}\right)^{2}}{\sum_{i=1}^{n}\left(Q_{i}-\bar{Q}\right)^{2}}
$$

where $n$ is the number of observed data; $Q_{i}$ and $\hat{Q}_{i}$ are the observed and forecasted values, respectively; $\bar{Q}_{i}$ and $\overline{\hat{Q}}_{i}$ are the averages of all observed and forecasted values, respectively.

\section{Case studies}

\subsection{Study area and data}

Two multipurpose Reservoirs, the Xinfengjiang and Guangzhao Reservoirs illustrated in Fig. 3, were 
selected as case studies. The Xinfengjiang Reservoir is located on the Xinfeng River, which is the largest tributary of the Dongjiang River, a second-level tributary of the Pearl River. With 336.1 MW of installed capacity and 13.896 billion $\mathrm{m}^{3}$ of storage volume as well as $5740 \mathrm{~km}^{2}$ of drainage area, the Xinfengjiang Reservoir is the largest Reservoir in the south of China. For the Xinfengjiang Reservoir, the primary goal is power generation. The Guangzhao Reservoir is located on the middle reaches of the Beipan River, which is a tributary of the Xijiang River, a second-level tributary of the Pearl River. With 1040MW of installed capacity and 3.245 billion $\mathrm{m}^{3}$ of storage volume as well as $13548 \mathrm{~km}^{2}$ of drainage area, the primary goal of the Guangzhao Reservoir is power generation. Hence, accurate monthly runoff forecasting is vital for these two reservoirs.

Monthly runoff series data from the Xinfengjiang and Guangzhao Reservoirs were retrieved for validating the proposed method. The monthly runoff hydrograph of the two reservoirs is depicted in Fig. 4, where the data for Xinfengjiang covers from 1943 to 2015 and the data for Guangzhao covers from 1956 to 2017. It can be seen that the monthly runoff for the two reservoirs varies over a wide range. For these two reservoirs, approximate $70 \%$ of the data was used for calibration and the remaining was used for validation.

\subsection{Decomposition results}

According to VMD, the key parameter of the number of mode should be predefined, which affects the decomposed results (Wen et al., 2019). To obtain satisfactory performance, the traditional EMD method was employed to ascertain the number of subsequences. The decomposed results for the Xinfengjiang and Guangzhao Reservoirs utilizing VMD and EMD are shown in Figs. 5 and 6, respectively. It can be found that there are significant differences in the acquired subcomponents for the two reservoirs, which indicate the variability of VMD and EMD in the aspect of extracting intrinsic information from original monthly runoff series.

\subsection{Input determination}

244 The selection of input variables that directly affects the forecast results, should be predetermined. As a statistical method, partial the partial autocorrelation function (PACF) can be employed to analysis and 
determine the input variables (Feng et al., 2020; He et al., 2019). In practice, the input variables are often determined by means of the PACF values that the pervious values are selected as inputs when all PACF values fall into the confidence interval. The PACF values for the original and decomposed subsequences of Xinfengjiang Reservoir data are shown in Figs. 7 and 8. On the basis of Figs. 7 and 8, the input variables for each sequences of Xinfengjiang and Guangzhao Reservoirs data can be determined. From Tables 1 and 2, it can be easily seen that the number of the input variables for the original and decomposed data are similar, but not always the same, indicating the complex and variable features of the data from the two reservoirs.

\section{Insert Here Fig. 7 \\ Insert Here Fig. 8 \\ Insert Here Table 1 \\ Insert Here Table 2}

\subsection{Model development}

To confirm the feasibility of the proposed method, five models were employed for comparison, namely back propagation neural networks (BPNN), support vector machine (SVM), LSTM, VMD-LSTM and EMD-LSTM models. The details of the models are stated as follows.

(1) BPNN model

The original monthly runoff data were used to calibrate the parameters of BPNN model. In this study, for the two reservoirs, three layers feedforward neural networks were employed, of which the input nodes were set based on PACF values of the original series and each sub-series, the output nodes were set as 1 and the hidden nodes were set by trial-and-error procedure.

(2) SVM model

The original monthly runoff data were used to calibrate the parameters of SVM model. In this study, the radial basis function was chosen as the kernel function, and the genetic algorithm was used to optimize the parameters of SVM model.

(3) LSTM model

Similar to the LMNN and SVM models, the original monthly runoff data were used to calibrate the parameters of LSTM model. The number of hidden layers is 2. For the LSTM model, the input nodes were set based on PACF values of the original series and each sub-series, the output nodes were set as 1 and the hidden units for each hidden layer were set by trial-and-error procedure. In addition, the 
hyperparameters, i.e. epoch and learning rate, were also set by trial-and-error procedure.

(4) VMD-LSTM and EMD-LSTM models

For the VMD-LSTM and EMD-LSTM models, there are there three main steps to be implemented. Firstly, the original monthly runoff data were decomposed into several subsequences using VMD or EMD. Secondly, the standard LSTM model was employed to simulate each subsequence and the input variables for each subsequence are listed in Tables 1 and 2. Finally, the results for each subsequence were aggregated as the final results.

\subsection{Forecast results}

\subsubsection{Results for the Xinfengjiang Reservoir}

According to the methods aforementioned, the original monthly runoff series and extracted subsequences were simulated. The detailed evaluation indexes of different models over the calibration and validation periods for the Xinfengjiang Reservoir is presented in Table 3. It can be intuitively found that compared with BPNN, SVM, LSTM, EMD-LSTM and VMD-LSTM models, the VMD-GWO-LSTM can yield the best results in terms of all the four evolution indexes both in calibration and validation periods. For instance, compared with the standalone BPNN model, the proposed hybrid VMD-GWO-LSTM model can respectively provide better forecast accuracy with decrement of $77.95 \%$ and $75.57 \%$ in terms of RMSE and MAPE and increment of $81.67 \%$ and $397.93 \%$ in terms of R and CE during the validation period. As can be seen in Table 3, the hybrid models, such as EMD-LSTM and VMD-LSTM, comprised of LSTM and decomposed methods outperform the standalone LSTM model in terms of all the four evolution indexes during the calibration and validation periods. For example, compared with the LSTM method, the VMD-LSTM model performs better with decrement of $72.06 \%$ and $57.51 \%$ in terms of RMSE and MAPE and increment of $52.66 \%$ and $154.96 \%$ in terms of R and CE during the validation period. In addition, Table 3 also reveals that the proposed hybrid model VMD-GWO-LSTM performs slightly better than the VMD-LSTM model in terms of the four measures both in the calibration and validation periods.

\section{Insert Here Table 3}

To detect the performance of tracing dynamic changes of the monthly runoff, a comparison of forecasted versus observed runoff data using BPNN, SVM, LSTM, EMD-LSTM, VMD-LSTM and VMD-GWO-LSTM for the Xinfengjiang Reservoir is depicted in Fig.9. On the whole, all of the models can simulate monthly runoff to some extent except for significant difference existed in peak flow 
prediction, indicating different models with different abilities in simulating peak runoff. To comprehend the performance of the models, the scatter diagrams for the Xinfengjiang Reservoir are presented in Fig.10, which shows less scatters with VMD-GWO-LSTM than the other five models and are consistent with the results in Table 3.

In addition, to assess the performance of the proposed hybrid mode in peak flow forecasting, peak flow estimates of different models over the validation period for the Xinfengjiang Reservoir can be proceeded by statistical analysis. As shown in Table 4, the absolute average of the relative error of the BPNN, SVM, LSTM, EMD-LSTM, VMD-LSTM and VMD-GWO-LSTM for forecasting the 21 peak flows are 38.9\%, $46.0 \%, 43.7 \%, 23.2 \%, 10.8 \%$ and $9.4 \%$, respectively. It can be easily concluded that in the aspect of peak flow forecast, the VMD-GWO-LSTM model can yield much better forecast accuracy than BPNN, SVM, LSTM and EMD-LSTM, and outperform slightly better forecast than the VMD-LSTM.

\subsubsection{Results for the Guangzhao Reservoir}

The statistics of different models over the calibration and validation periods for the Guangzhao Reservoir is shown in Table 5. It can be easily seen that the hybrid methods, namely, EMD-LSTM, VMDLSTM and VMD-GWO-LSTM display better performance than the standalone BPNN, SVM and LSTM. Furthermore, Table 5 also reveals that forecast accuracy of the LSTM model can be enhanced under the condition of optimized hyperparameters. For instance, compared to the SVM model, the VMD-LSTM can respectively provide better forecast accuracy with decrement of $59.06 \%$ and $65.66 \%$ in terms of RMSE and MAPE and increment of $31.73 \%$ and $80.51 \%$ in terms of R and CE during the validation period; compared to the VMD-LSTM model, the VMD-GWO-LSTM model can respectively provide better forecast accuracy with decrement of $36.13 \%$ and $21.39 \%$ in terms of RMSE and MAPE and increment of $2.61 \%$ and $5.34 \%$ in terms of R and CE during the validation period. Hence, it reconfirms that the proposed hybrid model is superior to the other models utilized in this study.

Insert Here Table 5

The forecast results of different models for the Guangzhao Reservoir during the validation phrase are drawn in Fig.11. It is clear from the hydrographs that the BPNN model having the worst performance of tracing dynamic changes of the monthly runoff and the rest models can afford satisfactory forecast results. 
The scatter diagrams for the Guangzhao Reservoir are plotted in Fig.10. It can be intuitively found that the VMD-GWO-LSTM model can offer the least forecast results among the six models and display the best performance with a trendline very near to the observed data line.

\section{Insert Here Fig. 11}

Insert Here Fig. 12

Table 6 lists the statistics of peak flow estimates of different models for the Guangzhao Reservoir during the validation period. From Table 6, the absolute average of the relative error of the BPNN, SVM, LSTM, EMD-LSTM, VMD-LSTM and VMD-GWO-LSTM models for forecasting the 18 peak flows are $31.4 \%, 33.2 \%, 31.7 \%, 15.8 \%, 7.6 \%$ and $6.2 \%$, respectively. Thus, in terms of peak flow forecast, the VMD-GWO-LSTM model can perform much better than the BPNN, SVM, LSTM and EMD-LSTM models, and slightly better than the VMD-LSTM model. As a consequence, the VMD-GWO-LSTM model is an efficient method for monthly runoff forecasting ascribing its superior performance over the comparable models during the validation period.

\section{Insert Here Table 6}

\subsection{Discussion}

According to the forecast results provided by BPNN, SVM and LSTM, it can be directly found that there are significant differences in terms of the four evolution indexes, demonstrating the importance of model selection and model parameter calibration. For the BPNN model, the gradient-based training algorithms have to face some drawbacks, such as over-fitting and local optimum (Wu and Chau, 2011). The ordinary SVM employing the structural risk-minimization principle can obtain good generalization performance. Nonetheless, the performance of SVM usually relies on the optimization algorithm to optimize the parameters and many studies can be referred in the literature (Feng et al., 2020b). As a deep learning algorithm, LSTM can overcome the gradient vanishing/exploding problem faced by traditional RNN and exhibit good generalization performance in hydrological time series prediction (Kratzert et al., 2018). Influenced by many factors such as human activities and climate changes, runoff usually contains multifrequency components (Niu et al., 2019). Hence, it is difficult to use a standalone prediction model to completely simulate runoff precisely because only one resolution component is used and the underlying multi-scale phenomena can't be unraveled. As decomposition methods, EMD and VMD are utilized to identify the multifrequency components to decrease the modelling difficulty. Therefore, the EMD-LSTM and VMD-LSTM models performed better than the standalone LSTM. Although many 
successful applications of LSTM have not involved how to optimize the hyperparameters, it is still worth paying much attrition to the hyperparameter optimization to enhance model performance and swarm intelligent algorithms (i.e., GWO) can be selected as possible solutions. Consequently, the proposed model VMD-GWO-LSTM outperformed the VMD-LSTM model.

The probable causes of VMD-GWO-LSTM superior to the comparable models can be generally attributed to the contribution of VMD decomposition and hyperparameters optimization based on GWO for LSTM. VMD can decompose the monthly runoff time series into several subsequences and ravel the underlying multi-scale phenomena implied in the monthly runoff time series. Each subsequence was simulated by LSTM with hyperparameters optimization conducted by GWO, which can identify the dynamic changes and decrease the modelling difficulty. Meanwhile, automatic optimization of hyperparameters of LSTM conquers the drawbacks of presetting parameters easily causing to lower forecast accuracy.

Although the feasibility of VMD-GWO-LSTM has been verified with monthly runoff data derived from two reservoirs, further research should be made in the future. It is necessary to involve new and excellent decomposition algorithms to enhance the quality of subsequences. Of course, more machine learning techniques should be investigated and verified to improve the single model forecast accuracy. Furthermore, the standard swarm optimization algorithms, for example GWO used in this study, should be modified to improve the quality of parametric optimization for the models.

In this study, a hybrid model, VMD-GWO-LSTM for short, has been proposed for forecasting monthly runoff. Firstly, the original monthly runoff data is decomposed into several subsequences. Secondly, each subsequence is simulated by a standalone LSTM model, of which the hyperparameters, including learning rate, epochs and hidden layer neurons, are optimized by GWO. Finally, all of the output of the standalone LSTM for each subsequence are aggregated as the final forecast results. Monthly runoff data derived from two reservoirs (Xinfengjiang and Guangzhao Reservoirs) located in China were employed to investigated the proposed hybrid model. In order to evaluate the model performance, four commonly used statistical evolution indexes were utilized and five models, namely BPNN, SVM, LSTM, EMDLSTM and VMD-LSTM, were used for comparison. The results indicated that the proposed model outperformed the five models in terms of all the four evolution indexes. Hence, the proposed method is feasible and promising for improving the forecasting accuracy of monthly runoff prediction. 


\section{Acknowledgements}

This paper is supported by the National Natural Science Foundation of China (51709109).

\section{Conflict of Interest}

The authors declare that they have no known competing financial interests or personal relationships that could have appeared to influence the work reported in this paper

\section{References}

Altan, A., Karasu, S., Zio, E., 2021. A new hybrid model for wind speed forecasting combining long short-term memory neural network, decomposition methods and grey wolf optimizer. Appl Soft Comput, 100. DOI:10.1016/j.asoc.2020.106996

ASCE-Task-Committee, 2000. Artificial neural networks in hydrology-II: Hydrological applications. J Hydrol Eng, 5(2): 124-137.

Colominas, M.A., Schlotthauer, G., Torres, M.E., 2014. Improved complete ensemble EMD: A suitable tool for biomedical signal processing. Biomed Signal Process Control, 14: 19-29. DOI:10.1016/j.bspc. 2014.06.009

Dakhlalla, A.O., Parajuli, P.B., 2016. Evaluation of the best management practices at the watershed scale to attenuate peak streamflow under climate change scenarios. Water Resour Manag, 30(3): 963-982. DOI:10.1007/s11269-015-1202-9

Dragomiretskiy, K., Zosso, D., 2014. Variational mode decomposition. IEEE Trans on Signal Process, 62(3): 531-544. DOI:10.1109/tsp.2013.2288675

ElSaid, A., El Jamiy, F., Higgins, J., Wild, B., Desell, T., 2018. Optimizing long short-term memory recurrent neural networks using ant colony optimization to predict turbine engine vibration. Appl Soft Comput, 73: 969-991. DOI:10.1016/j.asoc.2018.09.013

Fang, W., Huang, S., Ren, K., Huang, Q., Huang, G., Cheng, G., Li, K., 2019. Examining the applicability of different sampling techniques in the development of decomposition-based streamflow forecasting models. J Hydrol, 568: 534-550. DOI:10.1016/j.jhydrol.2018.11.020

Feng, Z.K., Niu, W.J., 2021. Hybrid artificial neural network and cooperation search algorithm for nonlinear river flow time series forecasting in humid and semi-humid regions. Knowl Based Syst, 211: 106580. DOI:10.1016/j.knosys.2020.106580

Feng, Z.K., Niu, W.J., Cheng, C.T., 2018. Optimization of hydropower reservoirs operation balancing generation benefit and ecological requirement with parallel multi-objective genetic algorithm. 
Feng, Z.K., Niu, W.J., Jiang, Z.Q., Qin, H., Song, Z.G., 2020a. Monthly operation optimization of cascade hydropower reservoirs with dynamic programming and latin hypercube sampling for dimensionality reduction. Water Resour Manag, 34(6): 2029-2041. DOI:10.1007/s11269-020-025450

Feng, Z.K., Niu, W.J., Tang, Z.Y., Jiang, Z.Q., Xu, Y., Liu, Y., Zhang, H.R., 2020b. Monthly runoff time series prediction by variational mode decomposition and support vector machine based on quantumbehaved particle swarm optimization. J Hydrol, 583. DOI:10.1016/j.jhydrol.2020.124627

Feng, Z.K., Niu, W.J., Tang, Z.Y., Xu, Y., Zhang, H.R., 2021. Evolutionary artificial intelligence model via cooperation search algorithm and extreme learning machine for multiple scales nonstationary hydrological time series prediction. J Hydrol, 595. DOI:10.1016/j.jhydrol.2021.126062

Hochreiter, S., Schmidhuber, J., 1997. Long Short-Term Memory. Neural Comput, 9(8): 1735-1780. DOI:10.1162/neco.1997.9.8.1735

Ji, C.-m., Zhou, T., Huang, H.-t., 2014. Operating rules derivation of Jinsha Reservoirs system with parameter calibrated support vector regression. Water Resour Manag, 28(9): 2435-2451. DOI:10.1007/s11269-014-0610-6

Kratzert, F., Klotz, D., Brenner, C., Schulz, K., Herrnegger, M., 2018. Rainfall-runoff modelling using long short-term memory (LSTM) networks. Hydrol Earth Syst Sci, 22(11): 6005-6022. DOI:10.5194/hess-22-6005-2018

Li, F.G., Ma, G.W., Chen, S.J., Huang, W.B., 2021. An ensemble modeling approach to forecast daily reservoir inflow using bidirectional long- and short-term memory (Bi-LSTM), variational mode decomposition (VMD), and energy entropy method. Water Resour Manag, 35(9): 2941-2963. DOI:10.1007/s11269-021-02879-3

Li, Y., Wu, H., Liu, H., 2018. Multi-step wind speed forecasting using EWT decomposition, LSTM principal computing, RELM subordinate computing and IEWT reconstruction. Energy Convers Manag, 167: 203-219. DOI:10.1016/j.enconman.2018.04.082

Liao, S.L., Li, G., Sun, Q.Y., Li, Z.F., 2016. Real-time correction of antecedent precipitation for the Xinanjiang model using the genetic algorithm. J Hydroinform, 18(5): 803-815. DOI:10.2166/hydro.2016.168

Liao, S.L., Liu, Z.W., Liu, B.X., Cheng, C.T., Jin, X.F., Zhao, Z.P., 2020. Multistep-ahead daily inflow 
forecasting using the ERA-Interim reanalysis data set based on gradient-boosting regression trees. Hydrol Earth Syst Sci, 24(5): 2343-2363. DOI:10.5194/hess-24-2343-2020

Liu, H., Mi, X.W., Li, Y.F., 2018. Smart multi-step deep learning model for wind speed forecasting based on variational mode decomposition, singular spectrum analysis, LSTM network and ELM. Energy Convers Manag, 159: 54-64. DOI:10.1016/j.enconman.2018.01.010 cyclic model with mutual information for hydrology forecasting: A Case study in the xixian basin. Adv Water Resour, 141: 10. DOI:10.1016/j.advwatres.2020.103622

Madsen, H., 2003. Parameter estimation in distributed hydrological catchment modelling using automatic calibration with multiple objectives. Adv Water Resour, 26(2): 205-216. DOI:10.1016/s0309-1708(02)00092-1

Mirjalili, S., Mirjalili, S.M., Lewis, A., 2014. Grey wolf optimizer. Adv Eng Softw, 69: 46-61. DOI:10.1016/j.advengsoft.2013.12.007

Nakisa, B., Rastgoo, M.N., Rakotonirainy, A., Maire, F., Chandran, V., 2018. Long short term memory hyperparameter optimization for a neural network based emotion recognition framework. IEEE Access, 6: 49325-49338. DOI:10.1109/access.2018.2868361

Ni, L., Wang, D., Singh, V.P., Wu, J., Wang, Y., Tao, Y., Zhang, J., 2020. Streamflow and rainfall forecasting by two long short-term memory-based models. J Hydrol, 583: 124296. DOI:10.1016/j.jhydrol.2019.124296

Niu, W.J., Feng, Z.K., Liu, S., Chen, Y.B., Xu, Y.S., Zhang, J., 2021. Multiple hydropower reservoirs operation by hyperbolic grey wolf optimizer based on elitism selection and adaptive mutation. Water Resour Manag, 35(2): 573-591. DOI:10.1007/s11269-020-02737-8

Niu, W.j., Feng, Z.k., Zeng, M., Feng, B.f., Min, Y.W., Cheng, C.T., Zhou, J.Z., 2019. Forecasting reservoir monthly runoff via ensemble empirical mode decomposition and extreme learning machine optimized by an improved gravitational search algorithm. Appl Soft Comput, 82: 105589. DOI:10.1016/j.asoc.2019.105589

Nourani, V., Alami, M.T., Aminfar, M.H., 2009. A combined neural-wavelet model for prediction of Ligvanchai watershed precipitation. Eng Appl of Arti Intell, 22(3): 466-472. DOI:10.1016/j.engappai.2008.09.003 

DOI:10.1016/j.eswa.2017.06.009

Roushangar, K., Ghasempour, R., Nourani, V., 2021. The potential of integrated hybrid pre-postprocessing techniques for short- to long-term drought forecasting. J Hydroinform, 23(1): 117-135. DOI:10.2166/hydro.2020.088

Şen, Z., 2021. Reservoirs for water supply under climate change impact-A Review. Water Resour Manag, 35(11): 3827-3843. DOI:10.1007/s11269-021-02925-0

Seo, Y., Kim, S., Kisi, O., Singh, V.P., 2015. Daily water level forecasting using wavelet decomposition and artificial intelligence techniques. J Hydrol, 520: 224-243. DOI:10.1016/j.jhydrol.2014.11.050

Shoaib, M., Shamseldin, A.Y., Khan, S., Khan, M.M., Khan, Z.M., Sultan, T., Melville, B.W., 2018. A comparative study of various hybrid wavelet feedforward neural network models for runoff forecasting. Water Resour Manag, 32(1): 83-103. DOI:10.1007/s11269-017-1796-1

Sibtain, M., Li, X.S., Bashir, H., Azam, M.I., 2021. A hybrid model for runoff prediction using variational mode decomposition and artificial neural network. Water Resour, 48(5): 701-712. DOI:10.1134/s0097807821050171

Tikhamarine, Y., Souag-Gamane, D., Ahmed, A.N., Kisi, O., El-Shafie, A., 2020. Improving artificial intelligence models accuracy for monthly streamflow forecasting using grey Wolf optimization (GWO) algorithm. J Hydrol, 582. DOI:10.1016/j.jhydrol.2019.124435

Wan, F., Wang, F.Q., Yuan, W.L., 2017. The reservoir runoff forecast with artificial neural network based on ant colony optimization. Appl Ecol and Environ Res, 15(4): 497-510. DOI:10.15666/aeer/1504_ 497510

Wang, W.C., Chau, K.W., Xu, D.M., Chen, X.Y., 2015. Improving forecasting accuracy of annual runoff time series using ARIMA based on EEMD decomposition. Water Resour Manag, 29(8): 2655-2675. DOI:10.1007/s11269-015-0962-6

Wang, X.J., Wang, Y.P., Yuan, P.X., Wang, L., Cheng, D.L., 2021. An adaptive daily runoff forecast model using VMD-LSTM-PSO hybrid approach. Hydrol Sci J, 66(9): 1488-1502. DOI:10.1080/02626667. 2021.1937631

Wang, Y.X., Liu, F.Y., Jiang, Z.S., He, S.L., Mo, Q.Y., 2017. Complex variational mode decomposition for signal processing applications. Mech Syst Signal Process, 86: 75-85. DOI:10.1016/j.ymssp. 2016.09.032 
516 Wu, C.L., Chau, K.W., 2011. Rainfall-runoff modeling using artificial neural network coupled with 517 singular spectrum analysis. J Hydr, 399(3-4): 394-409. DOI:10.1016/j.jhydrol.2011.01.017

518 Xu, B., Boyce, S.E., Zhang, Y., Liu, Q., Guo, L., Zhong, P.-A., 2017. Stochastic programming with a 519 joint chance constraint model for reservoir refill operation considering flood risk. J Water Resour 520 Plan Manag, 143(1): 04016067. DOI:doi:10.1061/(ASCE)WR.1943-5452.0000715

521 Yu, J., Wang, S., Xi, L., 2008. Evolving artificial neural networks using an improved PSO and DPSO. 522 Neurocomputing, 71(4): 1054-1060. DOI:10.1016/j.neucom.2007.10.013

523

524

525

526

527

528

529

530

531

532

533

Yuan, X.H., Chen, C., Lei, X.H., Yuan, Y.B., Adnan, R.M., 2018. Monthly runoff forecasting based on LSTM-ALO model. Stoch Environ Res Risk Assess, 32(8): 2199-2212. DOI:10.1007/s00477-018$1560-\mathrm{y}$

Zhang, M., Jiang, Z.N., Feng, K., 2017. Research on variational mode decomposition in rolling bearings fault diagnosis of the multistage centrifugal pump. Mech Syst Signal Process, 93: 460-493. DOI:10.1016/j.ymssp.2017.02.013

Zuo, G.G., Luo, J.G., Wang, N., Lian, Y.N., He, X.X., 2020. Decomposition ensemble model based on variational mode decomposition and long short-term memory for streamflow forecasting. J Hydrol, 585. DOI:10.1016/j.jhydrol.2020.124776 
535 Fig. 1 Schematic diagram of long short-term memory neural networks

536 Fig.2 The flowchart of VMD-GWO-LSTM for monthly runoff forecasting

537 Fig. 3 Location of the Xinfengjiang and Guangzhao Reservoirs in China

538 Fig. 4 Monthly runoff hydrograph of the Xinfengjiang and Guangzhao Reservoirs

539 Fig. 5 Decomposed results of monthly runoff data in Xinfengjiang Reservoirs

540 Fig. 6 Decomposed results of monthly runoff data in Guangzhao Reservoirs

541 Fig. 7 PACF values of each series from the Xinfengjiang Reservoir

542 Fig. 8 PACF values of each series from the Guangzhao Reservoir

543 Fig. 9 Comparison of the forecast results for the Xinfengjiang Reservoir during the validation period

544 Fig. 10 Comparison of scatter diagrams of forecast results for the Xinfengjiang Reservoir during the $545 \quad$ validation period

546 Fig. 11 Comparison of the forecast results for the Guangzhao Reservoir during the validation period

547 Fig. 12 Comparison of scatter diagrams of forecast results during the validation period for the

$548 \quad$ Guangzhao Reservoir 


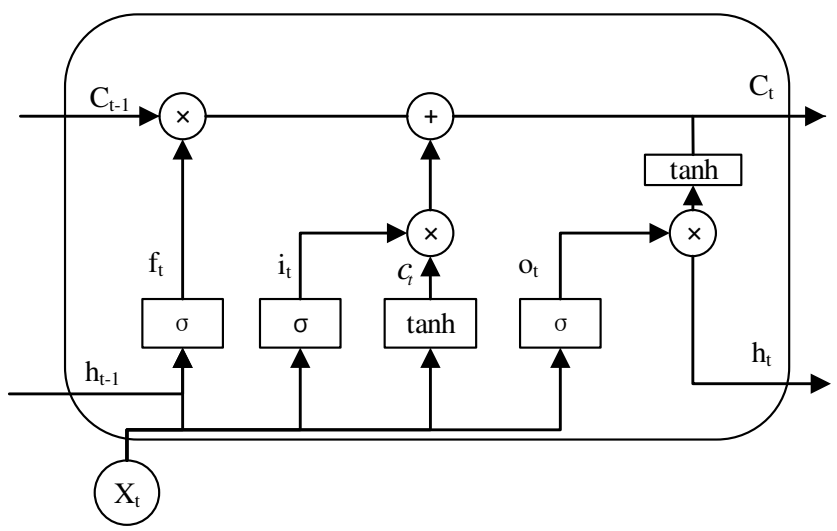

Fig. 1 Schematic diagram of long short-term memory neural networks 


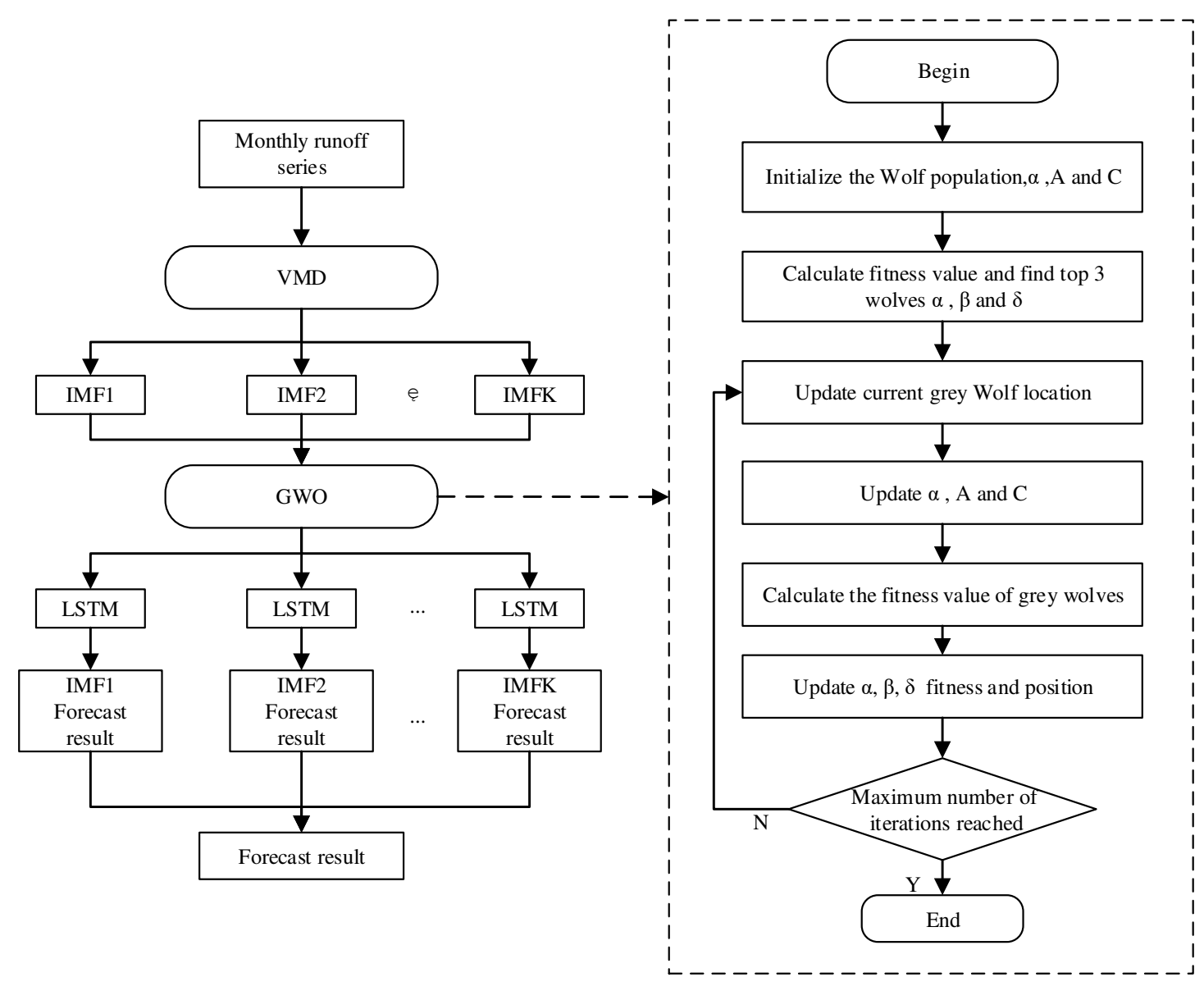

Fig. 2 The flowchart of VMD-GWO-LSTM for monthly runoff forecasting 


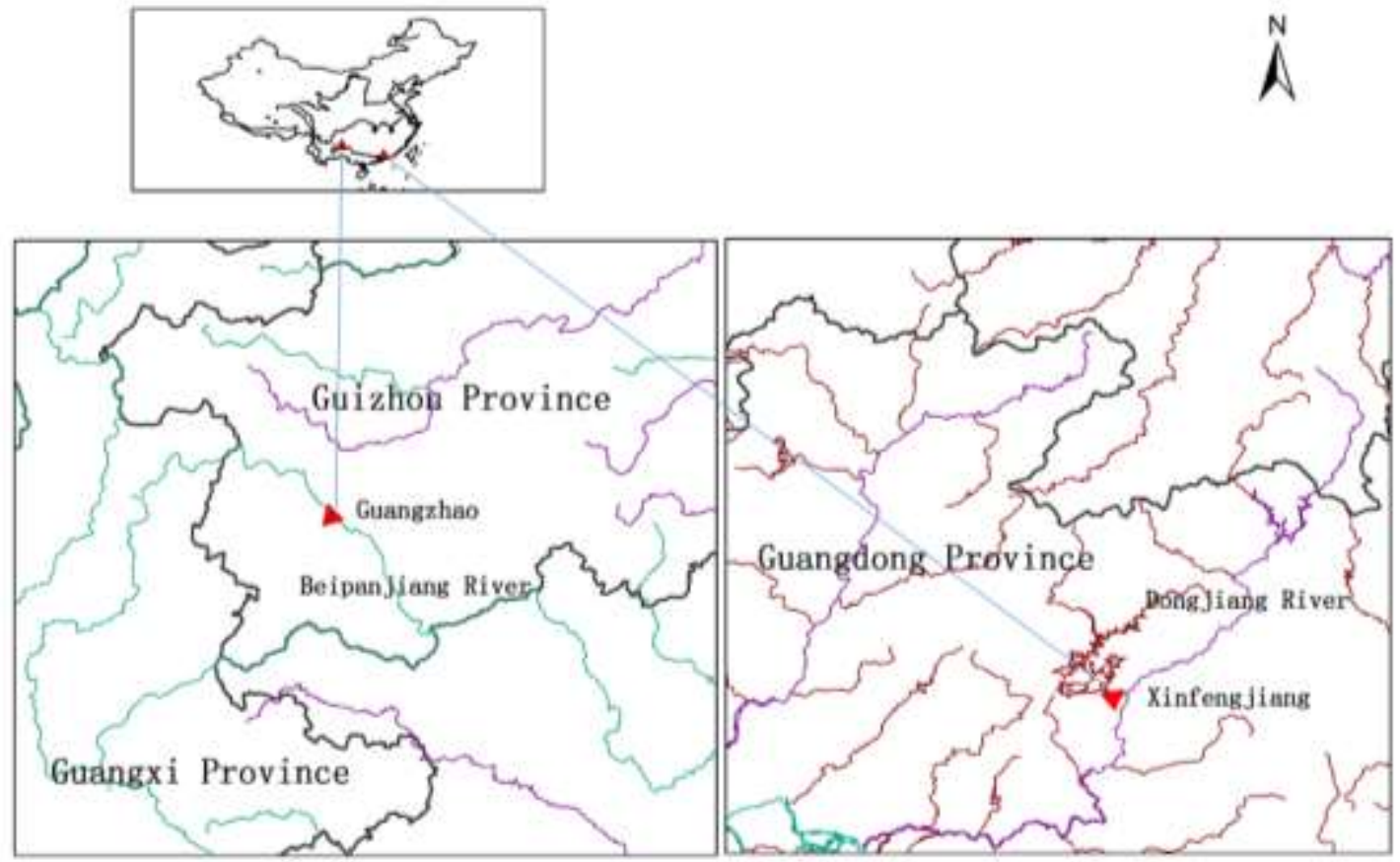

Fig. 3 Location of the Xinfengjiang and Guangzhao Reservoirs in China 


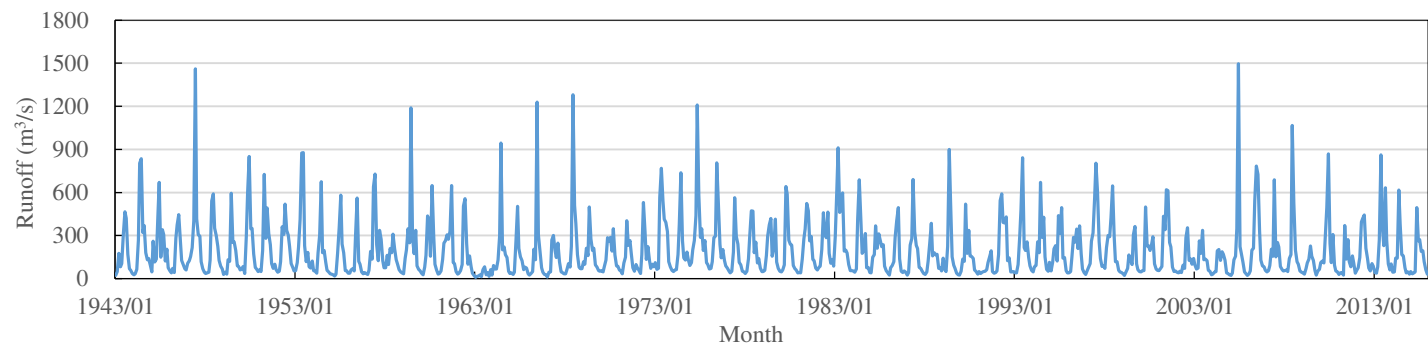

(a) Xinfengjiang

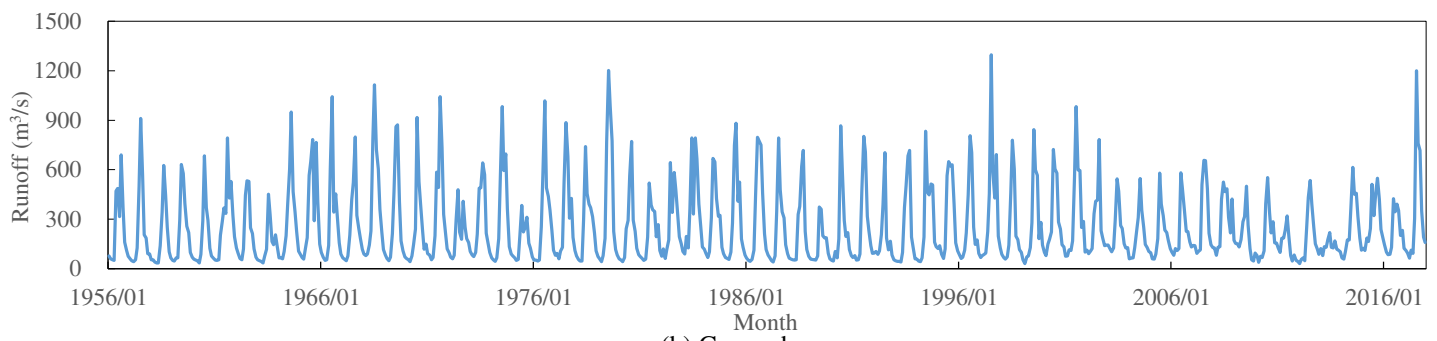

(b) Guangzhao

Fig. 4 Monthly runoff hydrograph of the Xinfengjiang and Guangzhao Reservoirs 

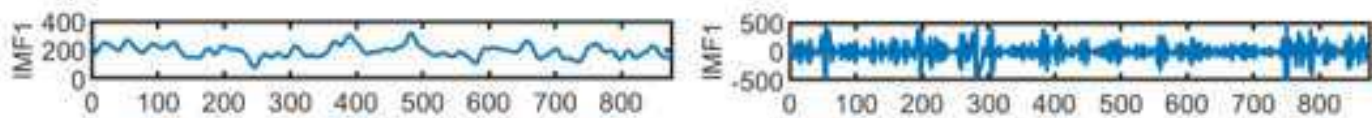

\& ${ }^{300}$ m

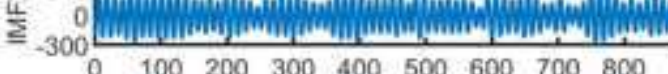

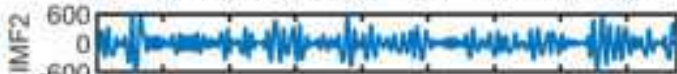

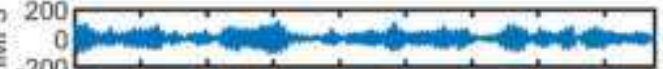

$-200 \frac{1}{0} \quad 100 \quad 200 \quad 300 \quad 400 \quad 500 \quad 600 \quad 700 \quad 800$

$\begin{array}{lllllllll}0 & 100 & 200 & 300 & 400 & 500 & 600 & 700 & 800\end{array}$

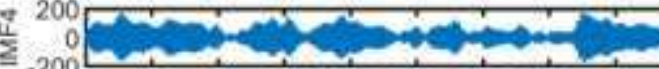

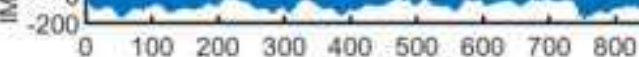

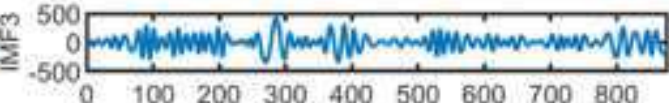

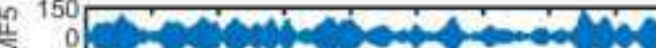

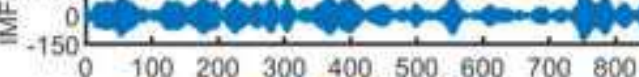

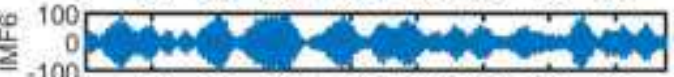

$=-100 \frac{0}{0} \quad 100 \quad 200 \quad 300 \quad 400 \quad 500 \quad 600 \quad 700 \quad 800$

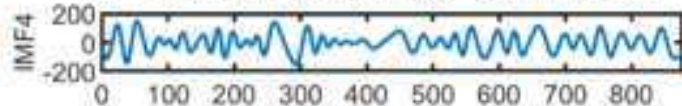

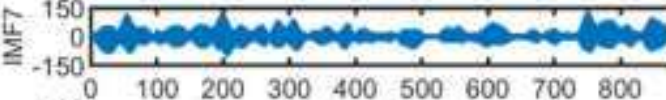
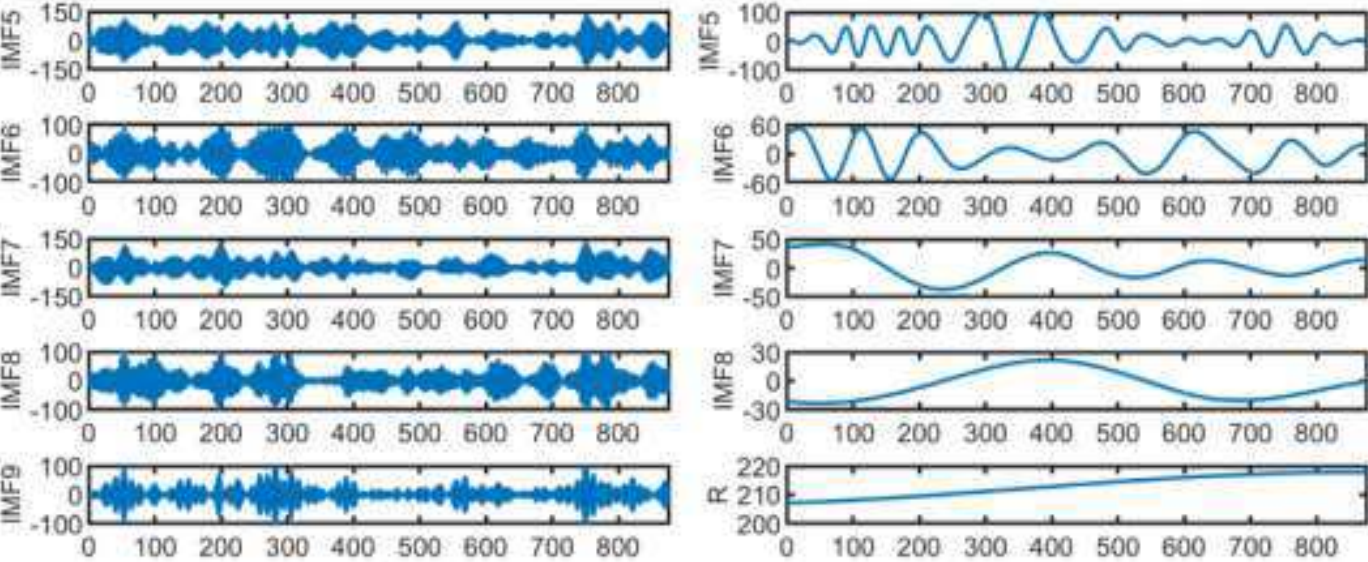

(a) VMD

(b) EMD

Fig. 5 Decomposed results of monthly runoff data in Xinfengjiang Reservoirs 
400
-300

$\underset{200}{300}$

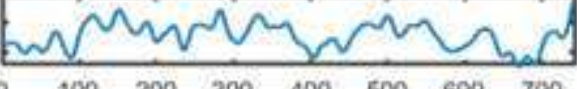

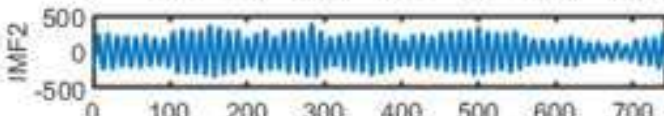
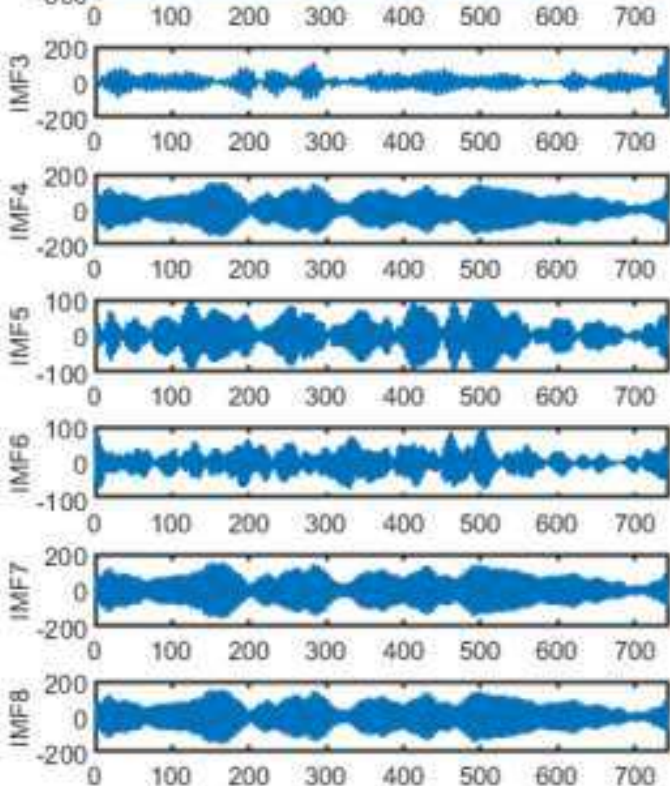

(a) VMD
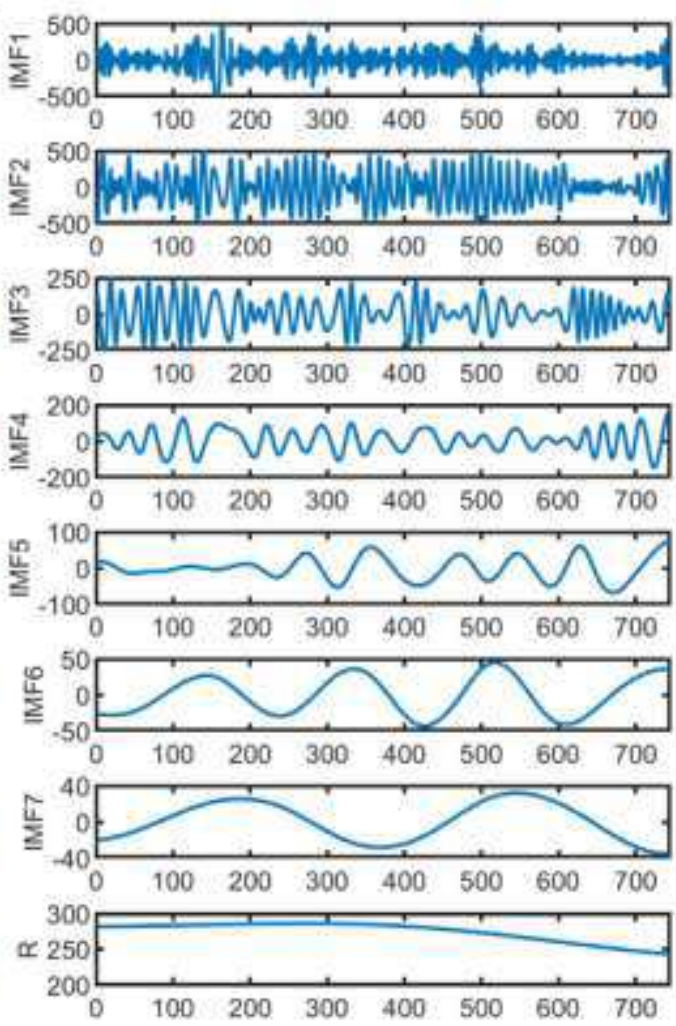

(b) EMD 


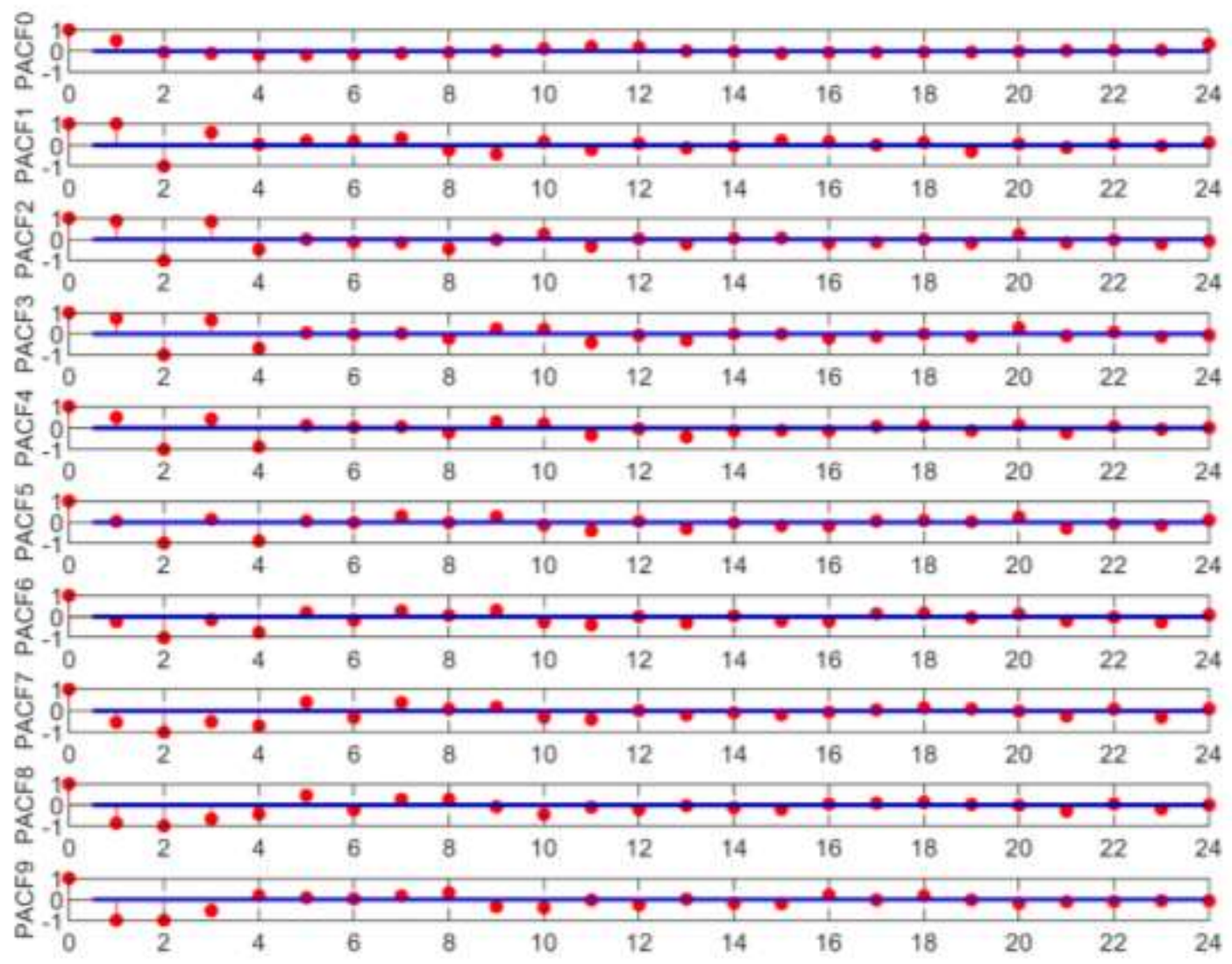

579

Fig. 7 PACF values of each series from the Xinfengjiang Reservoir 


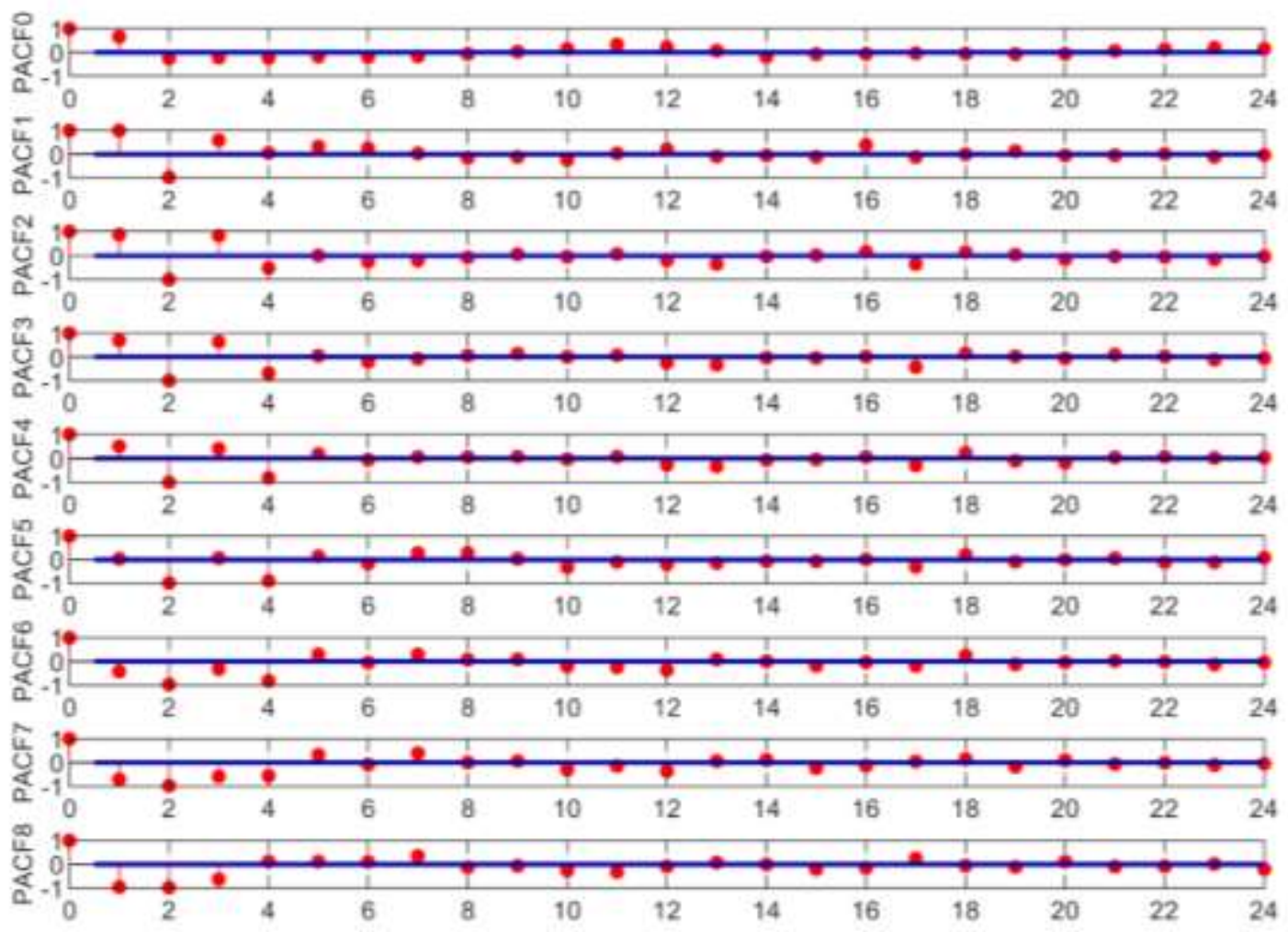

581 


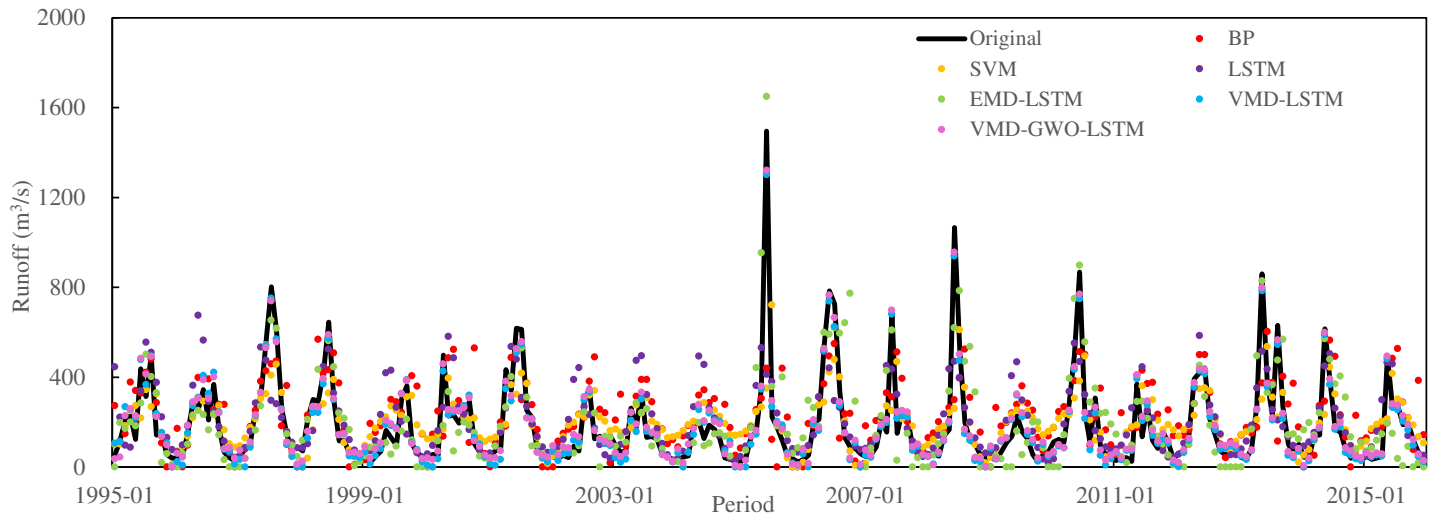

Fig.9 Comparison of the forecast results for the Xinfengjiang Reservoir during the validation period 

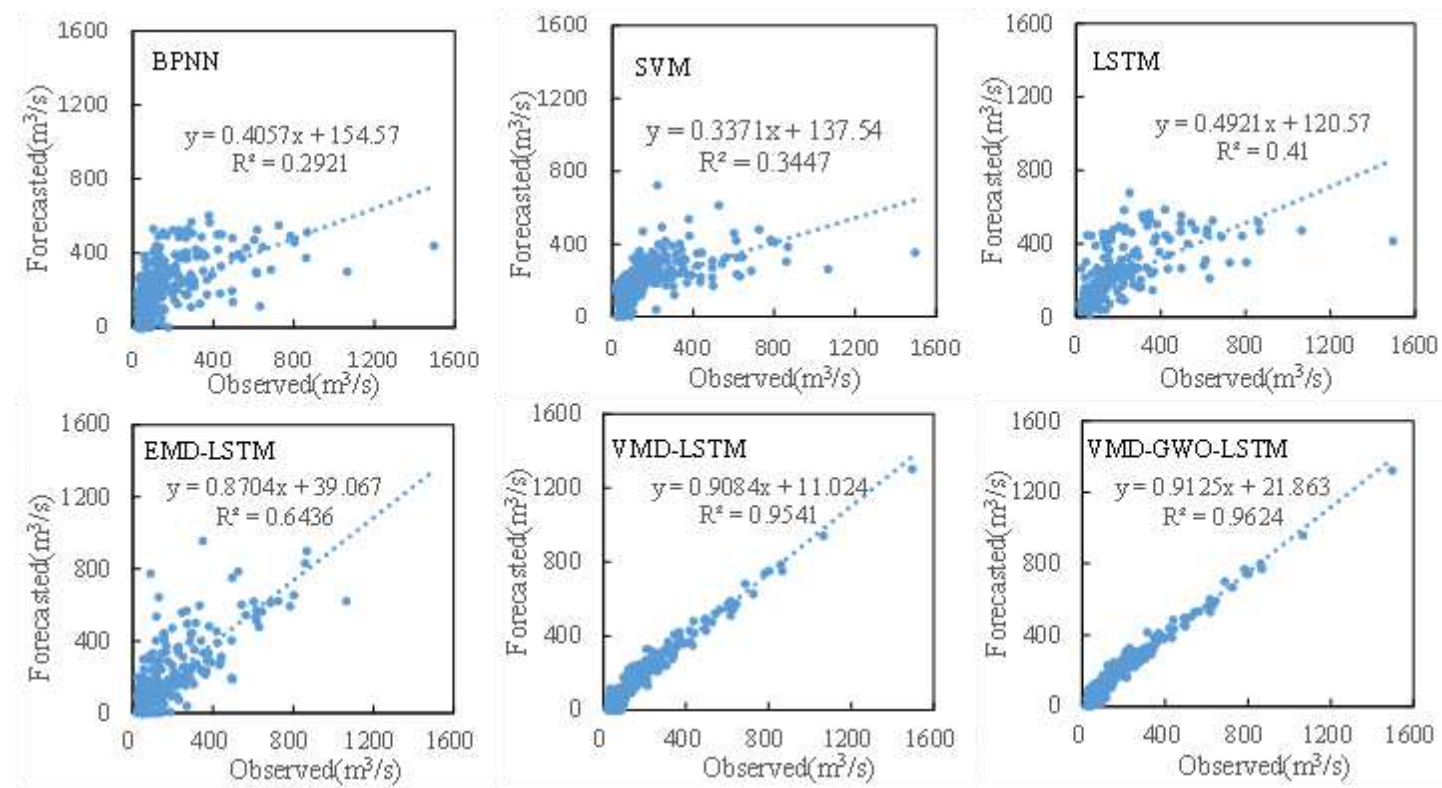

Fig. 10 Comparison of scatter diagrams of forecast results for the Xinfengjiang Reservoir during the 


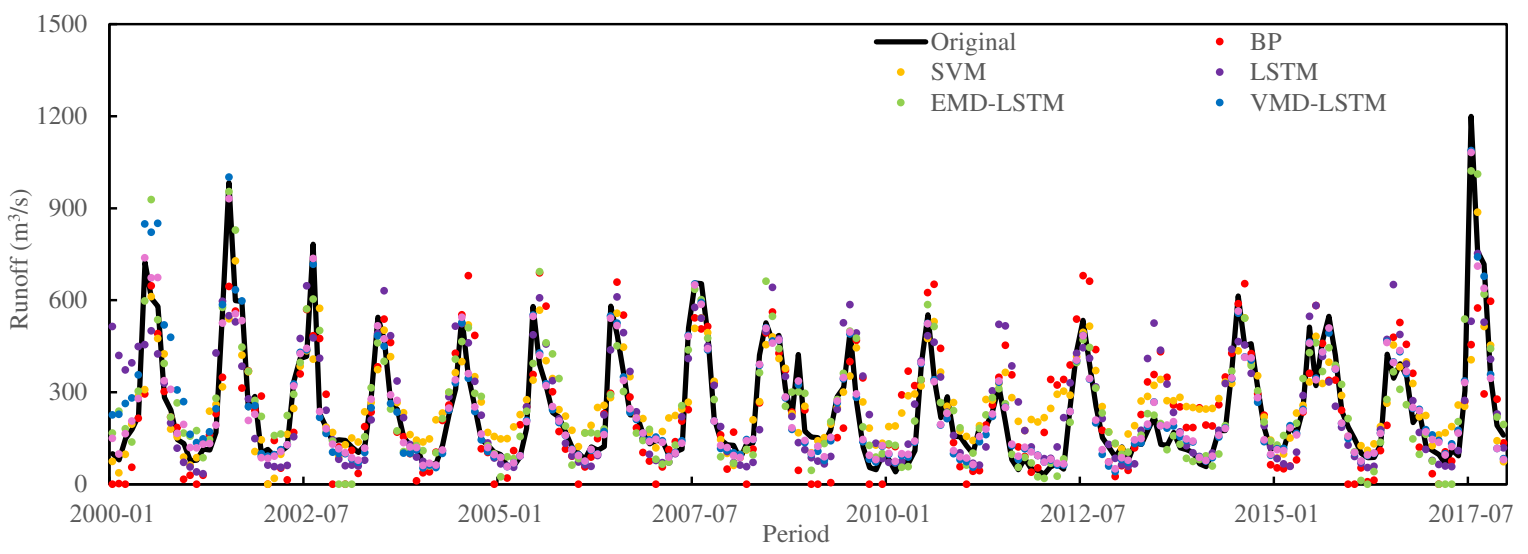

592 Fig.11 Comparison of the forecast results for the Guangzhao Reservoir during the validation period 

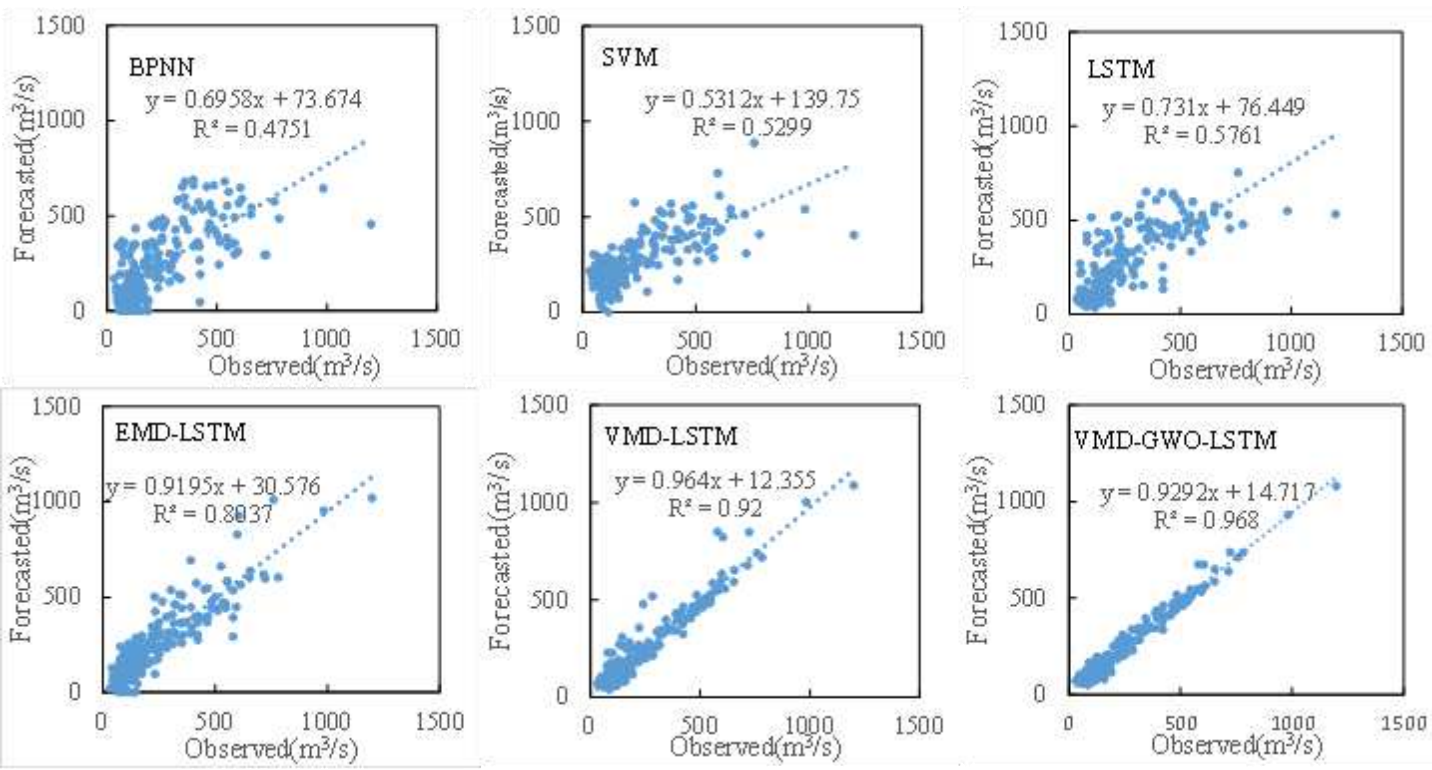

Fig. 12 Comparison of scatter diagrams of forecast results during the validation period for the 
599 Table 1 The selected input values of each series for the Xinfengiiang Reservoir monthly runoff data

600 Table 2 The selected input values of each series for the Guangzhao Reservoir monthly runoff data

601 Table 3 Comparison of evaluation indexes of different models for the Xinfengiiang Reservoir

602 Table 4 Peak flow estimates of different models for the Xinfengjiang Reservoir during the validation

603 period

604 Table 5 Comparison of evaluation indexes of different models for the Guangzhao Reservoir

605 Table 6 Peak flow estimates of different models for the Guangzhao Reservoir during the validation

606 period

607 
Table 1 The selected input values of each series for the Xinfengjiang Reservoir monthly runoff data

\begin{tabular}{|c|c|c|c|}
\hline No. & Series & Input variables & Numbers of input \\
\hline 1 & Original & $\mathrm{X}_{\mathrm{t}-1}, \mathrm{X}_{\mathrm{t}-2}, \mathrm{X}_{\mathrm{t}-3}, \mathrm{X}_{\mathrm{t}-4}, \mathrm{X}_{\mathrm{t}-5}, \mathrm{X}_{\mathrm{t}-6}, \mathrm{X}_{\mathrm{t}-7}, \mathrm{X}_{\mathrm{t}-8}$ & 8 \\
\hline 2 & $\mathrm{IMF}_{1}$ & $\mathrm{x}_{\mathrm{t}-1}, \mathrm{x}_{\mathrm{t}-2}, \mathrm{x}_{\mathrm{t}-3}$ & 3 \\
\hline 3 & $\mathrm{IMF}_{2}$ & $\mathrm{x}_{\mathrm{t}-1}, \mathrm{x}_{\mathrm{t}-2}, \mathrm{x}_{\mathrm{t}-3}, \mathrm{x}_{\mathrm{t}-4}$ & 4 \\
\hline 4 & $\mathrm{IMF}_{3}$ & $\mathrm{x}_{\mathrm{t}-1}, \mathrm{x}_{\mathrm{t}-2}, \mathrm{x}_{\mathrm{t}-3}, \mathrm{x}_{\mathrm{t}-4}$ & 4 \\
\hline 5 & $\mathrm{IMF}_{4}$ & $\mathrm{X}_{\mathrm{t}-1}, \mathrm{Xt}_{\mathrm{t}-2}, \mathrm{Xt}_{\mathrm{t}-3}, \mathrm{X}_{\mathrm{t}-4}$ & 4 \\
\hline 6 & $\mathrm{IMF}_{5}$ & $\mathrm{X}_{\mathrm{t}-1}, \mathrm{X}_{\mathrm{t}-2}, \mathrm{Xt}_{\mathrm{t}-3}, \mathrm{Xt}_{\mathrm{t}-4}$ & 4 \\
\hline 7 & $\mathrm{IMF}_{6}$ & $\mathrm{x}_{\mathrm{t}-1}, \mathrm{x}_{\mathrm{t}-2}, \mathrm{x}_{\mathrm{t}-3}, \mathrm{x}_{\mathrm{t}-4}, \mathrm{x}_{\mathrm{t}-5}, \mathrm{x}_{\mathrm{t}-6}, \mathrm{x}_{\mathrm{t}-7}$ & 7 \\
\hline 8 & $\mathrm{IMF}_{7}$ & $\mathrm{X}_{\mathrm{t}-1}, \mathrm{X}_{\mathrm{t}-2}, \mathrm{X}_{\mathrm{t}-3}, \mathrm{X}_{\mathrm{t}-4}, \mathrm{Xt}_{\mathrm{t}-5}, \mathrm{Xt}_{\mathrm{t}-6}, \mathrm{Xt}_{\mathrm{t}-7}$ & 7 \\
\hline 9 & $\mathrm{IMF}_{8}$ & $\mathrm{X}_{\mathrm{t}-1}, \mathrm{Xt}_{\mathrm{t}-2}, \mathrm{X}_{\mathrm{t}-3}, \mathrm{Xt}_{\mathrm{t}-4}, \mathrm{Xt}_{\mathrm{t}-5}, \mathrm{Xt}_{\mathrm{t}-6}, \mathrm{X}_{\mathrm{t}-7}, \mathrm{X}_{\mathrm{t}-8}$ & 8 \\
\hline 10 & $\mathrm{IMF}_{9}$ & $\mathrm{X}_{\mathrm{t}-1}, \mathrm{X}_{\mathrm{t}-2}, \mathrm{X}_{\mathrm{t}-3}, \mathrm{X}_{\mathrm{t}-4}$ & 4 \\
\hline
\end{tabular}




\begin{tabular}{|c|c|c|c|}
\hline No. & Series & Input variables & Numbers of input \\
\hline 1 & Original & $\mathrm{x}_{\mathrm{t}-1}, \mathrm{x}_{\mathrm{t}-2}, \mathrm{x}_{\mathrm{t}-3}, \mathrm{x}_{\mathrm{t}-4}, \mathrm{x}_{\mathrm{t}-5}, \mathrm{x}_{\mathrm{t}-6}, \mathrm{x}_{\mathrm{t}-7}$ & 7 \\
\hline 2 & $\mathrm{IMF}_{1}$ & $\mathrm{x}_{\mathrm{t}-1}, \mathrm{x}_{\mathrm{t}-2}, \mathrm{x}_{\mathrm{t}-3}$ & 3 \\
\hline 3 & $\mathrm{IMF}_{2}$ & $\mathrm{x}_{\mathrm{t}-1}, \mathrm{x}_{\mathrm{t}-2}, \mathrm{x}_{\mathrm{t}-3}, \mathrm{x}_{\mathrm{t}-4}$ & 4 \\
\hline 4 & $\mathrm{IMF}_{3}$ & $\mathrm{x}_{\mathrm{t}-1}, \mathrm{x}_{\mathrm{t}-2}, \mathrm{x}_{\mathrm{t}-3}, \mathrm{x}_{\mathrm{t}-4}$ & 4 \\
\hline 5 & $\mathrm{IMF}_{4}$ & $\mathrm{Xt}_{\mathrm{t}-1}, \mathrm{Xt}_{\mathrm{t}-2}, \mathrm{Xt}_{\mathrm{t}-3}, \mathrm{Xt}_{\mathrm{t}-4}, \mathrm{Xt}_{\mathrm{t}-5}$ & 5 \\
\hline 6 & $\mathrm{IMF}_{5}$ & $\mathrm{Xt}_{\mathrm{t}-1}, \mathrm{Xt}_{\mathrm{t}-2}, \mathrm{Xt}_{\mathrm{t}-3}, \mathrm{Xt}_{\mathrm{t}-4}, \mathrm{Xt}_{\mathrm{t}-5}, \mathrm{Xt}_{\mathrm{t}-6}, \mathrm{Xt}_{\mathrm{t}-7}, \mathrm{X}_{\mathrm{t}-8}$ & 8 \\
\hline 7 & $\mathrm{IMF}_{6}$ & $\mathrm{X}_{\mathrm{t}-1}, \mathrm{X}_{\mathrm{t}-2}, \mathrm{X}_{\mathrm{t}-3}, \mathrm{X}_{\mathrm{t}-4}, \mathrm{X}_{\mathrm{t}-5}$ & 5 \\
\hline 8 & $\mathrm{IMF}_{7}$ & $\mathrm{Xt}_{\mathrm{t}-1}, \mathrm{Xt}_{\mathrm{t}-2}, \mathrm{Xt}_{\mathrm{t}-3}, \mathrm{Xt}_{\mathrm{t}-4}, \mathrm{Xt}_{\mathrm{t}-5}$ & 5 \\
\hline 9 & $\mathrm{IMF}_{8}$ & $\mathrm{X}_{\mathrm{t}-1}, \mathrm{X}_{\mathrm{t}-2}, \mathrm{X}_{\mathrm{t}-3}, \mathrm{X}_{\mathrm{t}-4}$ & 4 \\
\hline
\end{tabular}

612

613 

Table 3 Comparison of evaluation indexes of different models for the Xinfengjiang Reservoir

\begin{tabular}{ccccccccc}
\hline \multirow{2}{*}{ Models } & \multicolumn{4}{c}{ Calibration } & \multicolumn{5}{c}{ Validation } \\
\cline { 2 - 9 } & RMSE & MAPE & R & CE & RMSE & MAPE & R & CE \\
\hline BPNN & 176.912 & 116.940 & 0.558 & 0.245 & 175.616 & 124.456 & 0.540 & 0.193 \\
SVM & 162.401 & 92.020 & 0.604 & 0.364 & 159.082 & 95.410 & 0.587 & 0.338 \\
LSTM & 143.215 & 73.108 & 0.713 & 0.505 & 154.706 & 76.900 & 0.640 & 0.373 \\
EMD-LSTM & 76.179 & 52.945 & 0.931 & 0.861 & 130.014 & 81.677 & 0.802 & 0.557 \\
VMD-LSTM & 39.524 & 30.710 & 0.984 & 0.962 & 43.224 & 32.676 & 0.977 & 0.951 \\
VMD-GWO-LSTM & 36.950 & 29.348 & 0.985 & 0.967 & 38.720 & 30.395 & 0.981 & 0.961 \\
\hline
\end{tabular}

615

616

617 

period

\begin{tabular}{|c|c|c|c|c|c|c|c|c|c|c|c|c|c|}
\hline \multirow{2}{*}{$\begin{array}{l}\text { Peak } \\
\text { NO. }\end{array}$} & \multirow{2}{*}{$\begin{array}{l}\text { Observed } \\
\text { peaks } \\
\left(\mathrm{m}^{3} / \mathrm{s}\right)\end{array}$} & \multirow{2}{*}{$\begin{array}{l}\text { BPNN } \\
\left(\mathrm{m}^{3} / \mathrm{s}\right)\end{array}$} & \multirow{2}{*}{$\begin{array}{l}\text { SVM } \\
\left(\mathrm{m}^{3} / \mathrm{s}\right)\end{array}$} & \multirow{2}{*}{$\begin{array}{l}\text { LSTM } \\
\left(\mathrm{m}^{3} / \mathrm{s}\right)\end{array}$} & \multirow{2}{*}{$\begin{array}{c}\text { EMD-LSTM } \\
\left(\mathrm{m}^{3} / \mathrm{s}\right)\end{array}$} & \multirow{2}{*}{$\begin{array}{l}\text { VMD-LSTM } \\
\left(\mathrm{m}^{3} / \mathrm{s}\right)\end{array}$} & \multirow{2}{*}{$\begin{array}{l}\text { VMD-GWO- } \\
\operatorname{LSTM}\left(\mathrm{m}^{3} / \mathrm{s}\right)\end{array}$} & \multicolumn{6}{|c|}{ Relative error $(\%)$} \\
\hline & & & & & & & & BPNN & SVM & LSTM & EMD-LSTM & VMD-LSTM & $\begin{array}{c}\text { VMD-GWO- } \\
\text { LSTM }\end{array}$ \\
\hline 1 & 494.0 & 481.1 & 269.0 & 510.0 & 402.1 & 493.5 & 492.3 & -2.6 & -45.6 & 3.2 & -18.6 & -0.1 & -0.4 \\
\hline 2 & 368.0 & 256.2 & 232.6 & 247.6 & 235.6 & 421.4 & 401.5 & -30.4 & -36.8 & -32.7 & -36.0 & 14.5 & 9.1 \\
\hline 3 & 803.0 & 458.8 & 408.5 & 296.9 & 653.2 & 751.2 & 739.4 & -42.9 & -49.1 & -63.0 & -18.7 & -6.5 & -7.9 \\
\hline 4 & 645.0 & 433.9 & 329.2 & 524.6 & 561.6 & 572.8 & 589.8 & -32.7 & -49.0 & -18.7 & -12.9 & -11.2 & -8.6 \\
\hline 5 & 362.0 & 385.8 & 311.7 & 145.4 & 212.9 & 388.7 & 385.6 & 6.6 & -13.9 & -59.8 & -41.2 & 7.4 & 6.5 \\
\hline 6 & 498.0 & 137.4 & 168.3 & 265.3 & 184.4 & 427.3 & 459.2 & -72.4 & -66.2 & -46.7 & -63.0 & -14.2 & -7.8 \\
\hline 7 & 618.0 & 526.7 & 324.4 & 479.4 & 508.7 & 508.8 & 526.4 & -14.8 & -47.5 & -22.4 & -17.7 & -17.7 & -14.8 \\
\hline 8 & 353.0 & 381.8 & 319.9 & 233.4 & 335.3 & 332.6 & 344.2 & 8.2 & -9.4 & -33.9 & -5.0 & -5.8 & -2.5 \\
\hline 9 & 336.0 & 389.9 & 239.5 & 495.3 & 243.1 & 303.6 & 332.8 & 16.0 & -28.7 & 47.4 & -27.7 & -9.7 & -0.9 \\
\hline 10 & 203.0 & 319.8 & 280.4 & 493.7 & 197.4 & 255.8 & 275.6 & 57.6 & 38.1 & 143.2 & -2.8 & 26.0 & 35.8 \\
\hline 11 & 1496.0 & 439.3 & 351.0 & 412.9 & 1649.7 & 1301.1 & 1321.0 & -70.6 & -76.5 & -72.4 & 10.3 & -13.0 & -11.7 \\
\hline 12 & 783.8 & 493.5 & 422.4 & 441.7 & 592.1 & 738.2 & 766.3 & -37.0 & -46.1 & -43.6 & -24.5 & -5.8 & -2.2 \\
\hline 13 & 687.5 & 311.7 & 251.4 & 436.1 & 610.4 & 680.8 & 698.2 & -54.7 & -63.4 & -36.6 & -11.2 & -1.0 & 1.6 \\
\hline 14 & 1066.0 & 299.6 & 260.5 & 470.4 & 619.8 & 940.2 & 956.8 & -71.9 & -75.6 & -55.9 & -41.9 & -11.8 & -10.2 \\
\hline 15 & 228.2 & 278.9 & 252.5 & 468.5 & 159.9 & 320.0 & 325.4 & 22.2 & 10.6 & 105.3 & -29.9 & 40.2 & 42.6 \\
\hline 16 & 867.5 & 512.7 & 382.7 & 469.6 & 897.9 & 748.6 & 769.1 & -40.9 & -55.9 & -45.9 & 3.5 & -13.7 & -11.3 \\
\hline 17 & 369.6 & 176.1 & 186.8 & 408.9 & 291.8 & 394.0 & 409.8 & -52.3 & -49.4 & 10.6 & -21.0 & 6.6 & 10.9 \\
\hline 18 & 442.3 & 500.5 & 352.2 & 424.0 & 313.4 & 414.8 & 433.3 & 13.2 & -20.4 & -4.1 & -29.1 & -6.2 & -2.0 \\
\hline 19 & 860.9 & 374.5 & 302.5 & 515.3 & 829.3 & 785.5 & 799.0 & -56.5 & -64.9 & -40.1 & -3.7 & -8.8 & -7.2 \\
\hline 20 & 616.2 & 292.6 & 233.3 & 450.7 & 570.9 & 589.3 & 599.8 & -52.5 & -62.1 & -26.9 & -7.4 & -4.4 & -2.7 \\
\hline 21 & 493.6 & 196.4 & 214.6 & 465.0 & 195.6 & 482.8 & 493.6 & -60.2 & -56.5 & -5.8 & -60.4 & -2.2 & 0.0 \\
\hline \multicolumn{8}{|c|}{ Average (absolute) } & 38.9 & 46.0 & 43.7 & 23.2 & 10.8 & 9.4 \\
\hline
\end{tabular}


Table 5 Comparison of evaluation indexes of different models for the Guangzhao Reservoir

\begin{tabular}{ccccccccc}
\hline \multirow{2}{*}{ Models } & \multicolumn{4}{c}{ Calibration } & \multicolumn{5}{c}{ Validation } \\
\cline { 2 - 9 } & RMSE & MAPE & R & CE & RMSE & MAPE & R & CE \\
\hline BPNN & 162.371 & 73.088 & 0.762 & 0.570 & 148.575 & 67.225 & 0.689 & 0.373 \\
SVM & 157.653 & 69.643 & 0.771 & 0.595 & 131.621 & 66.394 & 0.728 & 0.508 \\
LSTM & 134.204 & 32.806 & 0.845 & 0.706 & 128.575 & 50.008 & 0.759 & 0.530 \\
EMD-LSTM & 105.202 & 55.066 & 0.941 & 0.884 & 87.303 & 36.484 & 0.897 & 0.783 \\
VMD-LSTM & 39.146 & 19.660 & 0.989 & 0.975 & 53.880 & 22.798 & 0.959 & 0.917 \\
VMD-GWO-LSTM & 37.987 & 17.572 & 0.990 & 0.976 & 34.415 & 17.921 & 0.984 & 0.966 \\
\hline
\end{tabular}

623

624 
Table 6 Peak flow estimates of different models for the Guangzhao Reservoir during the validation period

\begin{tabular}{|c|c|c|c|c|c|c|c|c|c|c|c|c|c|}
\hline \multirow[b]{2}{*}{ Peak NO. } & \multirow{2}{*}{$\begin{array}{l}\text { Observed } \\
\text { peaks } \\
\left(\mathrm{m}^{3} / \mathrm{s}\right)\end{array}$} & \multirow{2}{*}{$\begin{array}{l}\text { BPNN } \\
\left(\mathrm{m}^{3} / \mathrm{s}\right)\end{array}$} & \multirow{2}{*}{$\begin{array}{l}\text { SVM } \\
\left(\mathrm{m}^{3} / \mathrm{s}\right)\end{array}$} & \multirow{2}{*}{$\begin{array}{l}\text { LSTM } \\
\left(\mathrm{m}^{3} / \mathrm{s}\right)\end{array}$} & \multirow{2}{*}{$\begin{array}{l}\text { EMD- } \\
\text { LSTM } \\
\left(\mathrm{m}^{3} / \mathrm{s}\right)\end{array}$} & \multirow{2}{*}{$\begin{array}{l}\text { VMD- } \\
\text { LSTM } \\
\left(\mathrm{m}^{3} / \mathrm{s}\right)\end{array}$} & \multirow{2}{*}{$\begin{array}{l}\text { VMD-GWO- } \\
\text { LSTM }\left(\mathrm{m}^{3} / \mathrm{s}\right)\end{array}$} & \multicolumn{6}{|c|}{ Relative error(\%) } \\
\hline & & & & & & & & BP & SVM & LSTM & EMD-LSTM & VMD-LSTM & $\begin{array}{l}\text { VMD-GWO- } \\
\text { LSTM }\end{array}$ \\
\hline 1 & 723.5 & 293.8 & 308.1 & 454.3 & 597.1 & 848.7 & 738.2 & -59.4 & -57.4 & -37.2 & -17.5 & 17.3 & 2.0 \\
\hline 2 & 983.3 & 644.1 & 539.3 & 548.6 & 953.6 & 1001.5 & 931.1 & -34.5 & -45.2 & -44.2 & -3.0 & 1.9 & -5.3 \\
\hline 3 & 782.6 & 484.0 & 407.5 & 477.1 & 603.5 & 717.1 & 736.4 & -38.1 & -47.9 & -39.0 & -22.9 & -8.4 & -5.9 \\
\hline 4 & 544.8 & 382.6 & 373.4 & 490.3 & 460.7 & 483.8 & 515.7 & -29.8 & -31.5 & -10.0 & -15.4 & -11.2 & -5.3 \\
\hline 5 & 546.4 & 552.6 & 399.6 & 523.2 & 465.2 & 525.7 & 544.5 & 1.1 & -26.9 & -4.3 & -14.9 & -3.8 & -0.4 \\
\hline 6 & 580.2 & 357.1 & 339.5 & 487.2 & 393.0 & 551.0 & 546.7 & -38.5 & -41.5 & -16.0 & -32.3 & -5.0 & -5.8 \\
\hline 7 & 580.5 & 295.9 & 282.2 & 437.2 & 291.8 & 547.9 & 541.0 & -49.0 & -51.4 & -24.7 & -49.7 & -5.6 & -6.8 \\
\hline 8 & 656.2 & 542.0 & 508.2 & 576.5 & 635.7 & 653.0 & 649.6 & -17.4 & -22.6 & -12.2 & -3.1 & -0.5 & -1.0 \\
\hline 9 & 526.0 & 492.7 & 454.3 & 497.9 & 661.7 & 510.0 & 508.3 & -6.3 & -13.6 & -5.4 & 25.8 & -3.1 & -3.4 \\
\hline 10 & 500.2 & 399.0 & 352.0 & 585.4 & 498.6 & 489.4 & 495.6 & -20.2 & -29.6 & 17.0 & -0.3 & -2.1 & -0.9 \\
\hline 11 & 552.7 & 624.3 & 474.3 & 483.4 & 584.9 & 523.9 & 523.3 & 13.0 & -14.2 & -12.5 & 5.8 & -5.2 & -5.3 \\
\hline 12 & 320.4 & 347.6 & 327.5 & 521.3 & 311.4 & 332.4 & 336.4 & 8.5 & 2.2 & 62.7 & -2.8 & 3.7 & 5.0 \\
\hline 13 & 535.0 & 679.8 & 495.1 & 445.0 & 471.0 & 484.7 & 483.7 & 27.1 & -7.5 & -16.8 & -12.0 & -9.4 & -9.6 \\
\hline 14 & 219.9 & 357.1 & 321.9 & 524.8 & 224.6 & 267.4 & 266.9 & 62.4 & 46.4 & 138.6 & 2.1 & 21.6 & 21.4 \\
\hline 15 & 614.1 & 588.4 & 434.5 & 464.8 & 563.2 & 556.8 & 564.1 & -4.2 & -29.2 & -24.3 & -8.3 & -9.3 & -8.1 \\
\hline 16 & 548.0 & 338.2 & 397.4 & 333.6 & 444.7 & 493.3 & 508.5 & -38.3 & -27.5 & -39.1 & -18.8 & -10.0 & -7.2 \\
\hline 17 & 423.9 & 191.3 & 263.8 & 471.5 & 275.8 & 465.2 & 461.9 & -54.9 & -37.8 & 11.2 & -34.9 & 9.7 & 9.0 \\
\hline 18 & 1199.6 & 454.5 & 405.7 & 531.1 & 1021.1 & 1087.9 & 1081.4 & -62.1 & -66.2 & -55.7 & -14.9 & -9.3 & -9.9 \\
\hline & & & Avera & rage (abso & llute) & & & 31.4 & 33.2 & 31.7 & 15.8 & 7.6 & 6.2 \\
\hline
\end{tabular}

